\title{
Current status of imaging biomarkers predicting the biological nature of hepatocellular carcinoma
}

\author{
Norihide Yoneda ${ }^{1} \cdot$ Osamu Matsui $^{1} \cdot$ Satoshi Kobayashi ${ }^{2} \cdot$ Azusa Kitao $^{1} \cdot$ Kazuto Kozaka $^{1} \cdot$ Dai Inoue $^{1}$. \\ Kotaro Yoshida ${ }^{1} \cdot$ Tetsuya Minami $^{3} \cdot$ Wataru Koda $^{1} \cdot$ Toshifumi Gabata $^{1}$
}

Received: 12 November 2018 / Accepted: 21 January 2019 / Published online: 2 February 2019

(c) Japan Radiological Society 2019

\begin{abstract}
Hepatocellular carcinoma (HCC) is heterogeneous in terms of its biological nature. Various factors related to its biological nature, including size, multifocality, macroscopic morphology, grade of differentiation, macro/microvascular invasion, bile duct invasion, intra-tumoral fat and molecular factors, and their value as prognostic imaging biomarkers have been reported. And recently, genome-based molecular HCC classification correlated with clinical outcome has been elucidated. The imaging biomarkers suggesting a less aggressive nature of HCC are smaller size, solitary tumor, smooth margin suggesting small nodular type with indistinct margin and simple nodular type with distinct margin, capsule, imaging biomarkers predicting early or well-differentiated grade, intra-tumoral fat detection, and low fluorodeoxyglucose (FDG) accumulation. The imaging biomarkers suggesting an aggressive HCC nature are larger size, multifocality, non-smooth margin suggesting simple nodular type with extranodular growth, confluent multinodular, and infiltrative type, imaging biomarkers predicting poor differentiation, macrovascular tumor thrombus, predicting microvascular invasion imaging biomarkers, bile duct dilatation or tumor thrombus, and high FDG accumulation. In the genome-based molecular classification, CTNNB-1 mutated HCC shows a less aggressive nature, while CK19/EpCAM positive HCC and macrotrabecular massive HCC show an aggressive one. Better understanding of these imaging biomarkers can contribute to devising more appropriate treatment plans for HCC.
\end{abstract}

Keywords Hepatocellular carcinoma · Biological nature $\cdot$ Imaging biomarkers · Genome-based molecular HCC classification

\begin{tabular}{|c|c|c|c|c|}
\hline \multicolumn{3}{|c|}{ Abbreviations } & SN-EG & Simple nodular type with extranodular \\
\hline \multicolumn{2}{|c|}{$\mathrm{HCC}$} & Hepatocellular carcinoma & & growth \\
\hline \multicolumn{2}{|c|}{ T1WI } & T1 weighted image & $\mathrm{CMN}$ & Confluent multinodular type \\
\hline \multicolumn{2}{|c|}{ MRI } & Magnetic resonance imaging & HB phase & Hepatobiliary phase \\
\hline \multicolumn{2}{|c|}{ T2WI } & T2 weighted image & $\mathrm{DN}$ & Dysplastic nodule \\
\hline \multicolumn{2}{|c|}{$\mathrm{IM}$} & Intrahepatic metastases & $\mathrm{ADC}$ & Apparent diffusion coefficient \\
\hline \multicolumn{2}{|c|}{ SN-IM } & Small nodular type with indistinct margin & DWI & Diffusion weighted image \\
\hline \multicolumn{2}{|c|}{ SN-DM } & Simple nodular type with distinct margin & FDG PET & $\begin{array}{l}\text { Fluorodeoxyglucose positron emission } \\
\text { tomography }\end{array}$ \\
\hline & & & OATP & Organic anion-transporting polypeptide \\
\hline & & & SPIO & Superparamagnetic iron oxide \\
\hline \multirow[t]{2}{*}{$\triangle$} & \multirow{2}{*}{\multicolumn{2}{|c|}{$\begin{array}{l}\text { Norihide Yoneda } \\
\text { noritiny@staff.kanazawa-u.ac.jp }\end{array}$}} & $\mathrm{HK}$ & Hexokinase \\
\hline & & & GLUT1 & Glucose transporter 1 \\
\hline \multirow[t]{3}{*}{1} & Depar & t of Radiology, Kanazawa University Graduate & G6Pase & Glucose-6-phosphatase \\
\hline & Schoo & Medical Sciences, 13-1 Takaramachi, Kanazawa, & PPV & Positive predictive value \\
\hline & Ishika & 20-8640, Japan & NPV & Negative predictive value \\
\hline \multirow[t]{3}{*}{2} & Depar & t of Quantum Medical Imaging, Kanazawa & SUV & Standardized uptake values \\
\hline & Unive & Graduate School of Medical Sciences, 13-1 & AFP & $\alpha$-Fetoprotein \\
\hline & Takars & ai, Kanazawa, Ishikawa 920-8640, Japan & PIVKA-II & Protein induced by Vitamin $\mathrm{K}$ absence or \\
\hline 3 & $\begin{array}{l}\text { Depar } \\
\text { Daigal }\end{array}$ & $\begin{array}{l}\text { f Radiology, Kanazawa Medical University, 1-1 } \\
\text { ninada, Ishikawa 920-0293, Japan }\end{array}$ & & antagonists-II \\
\hline
\end{tabular}




$\begin{array}{ll}\text { HNF } & \text { Hepatocyte nuclear factor (HNF) } \\ \text { EMT } & \text { Epithelial-mesenchymal transformation } \\ \text { CK } & \text { Cytokeratin } \\ \text { EPCAM } & \text { Epithelial cell adhesion molecule } \\ \text { CTNNB1 } & \text { Catenin beta 1 } \\ \text { MTM-HCC } & \text { Macrotrabecular massive HCC } \\ \text { SH-HCC } & \text { Steatohepatitic HCC }\end{array}$

\section{Introduction}

Hepatocellular carcinoma (HCC) is heterogeneous in terms of its biological behavior. The biological properties of $\mathrm{HCC}$ correlate well with prognosis. In other words, some HCCs have less aggressive biological natures with better prognosis, while others have aggressive biological natures with worse prognosis. Because the diagnosis of HCC is often made solely by imaging in the clinical setting, imaging plays an important role in the diagnosis of HCC and is also useful for predicting the biological properties of HCC.

Various factors related to the biological nature of HCC, such as size [1], multifocality [1], macroscopic morphology [2-4], grade of differentiation of cancer cells [5, 6], macro/ microvascular invasion [7-9], bile duct invasion [10], intratumoral fat [11] and molecular factors [12, 13], and their value as prognostic imaging biomarkers have been reported. Table 1 shows the various factors related to the biological nature of HCC and their imaging biomarkers. Information about the biological nature of HCC is important to determine the optimal therapeutic strategy. Therefore, an understanding of the imaging biomarkers predicting the biological nature of HCC is essential.

Recently, a genome-based HCC classification [14-16] has been elucidated and proven to be correlated with the clinical outcome. In addition, distinct histological variants are associated with specific molecular subclasses $[15,16]$. This classification has the possibility of contributing to future target therapy and personalized care.

In this review, we discuss the various imaging biomarkers predicting the biological nature of $\mathrm{HCC}$ and review the genome-based HCC classification correlated with imaging and its possibility as an imaging biomarker for future perspective.

\section{Size}

The size of HCC is an important prognostic factor [1]. In patients with large HCC, long-term prognosis is generally poor. Larger HCCs $(>5 \mathrm{~cm})$ have a higher incidence of macro/microscopic vascular invasion, and more advanced histologic grade [17]. On the other hand, small HCCs $(<2 \mathrm{~cm})$ consist of two subtypes: early HCC and classic small HCC [18]. Early HCC has the following pathological characteristics: consists of well-differentiated tumor cells [19], shows stromal invasion [20], grows by replacing the surrounding liver parenchyma [19] and presents an indistinct margin. Radiologically, this early HCC tends to show an atypical enhancement pattern of HCC with a lack of arterial hyper enhancement [18] (Fig. 1). Decreased intra-nodular portal supply, hyperintensity on T1-weighted images (T1WI) of magnetic resonance imaging (MRI), and isointensity or hypointensity on T2-weighted images (T2WI) are also imaging features of early HCC (Fig. 1). On the other hand, classic small HCC shows moderate differentiation (about 80\%), both well- and moderately differentiated components (20\%) with a distinct margin (expanding growth) [20], frequent nodule in nodule pattern and the absence of portal tracts

Table 1 Various factors related with biological nature of $\mathrm{HCC}$ and their imaging biomarkers

\begin{tabular}{ll}
\hline Factors related with biological nature of HCC & Imaging biomarkers \\
\hline Size & Size \\
Multifocality & Multifocality \\
Macroscopic morphology & Shape/Tumor margin/Capsule \\
Grade of differentiation of cancer cells & Intra-tumoral blood supply/ Gadoxetic acid uptake/ Kupffer cell imaging/ T1, T2 \\
& signal intensity/ DWI (ADC) \\
Macrovascular invasion & Macrovascular tumor thrombus \\
Microvascular invasion (MVI) & Tumor margin/ Peritumoral enhancement/ Peritumoral hypointensity on HB \\
& phase/ ADC/ Size/ Arterial enhancement/ FDG uptake/ capsule/ Multifocality \\
Bile duct invasion & Bile duct dilatation or tumor thrombus \\
Intra-tumoral fat & Intra-tumoral fat detection \\
Molecular factors & FDG uptake/HB phase hyperintense HCC \\
\hline Future prospects &
\end{tabular}

Genome-based molecular classification and imaging features

(CK19 positive HCC, EpCAM positive HCC, MTM-HCC, Steatohepatitic HCC, CTNNB-1 mutated HCC) 

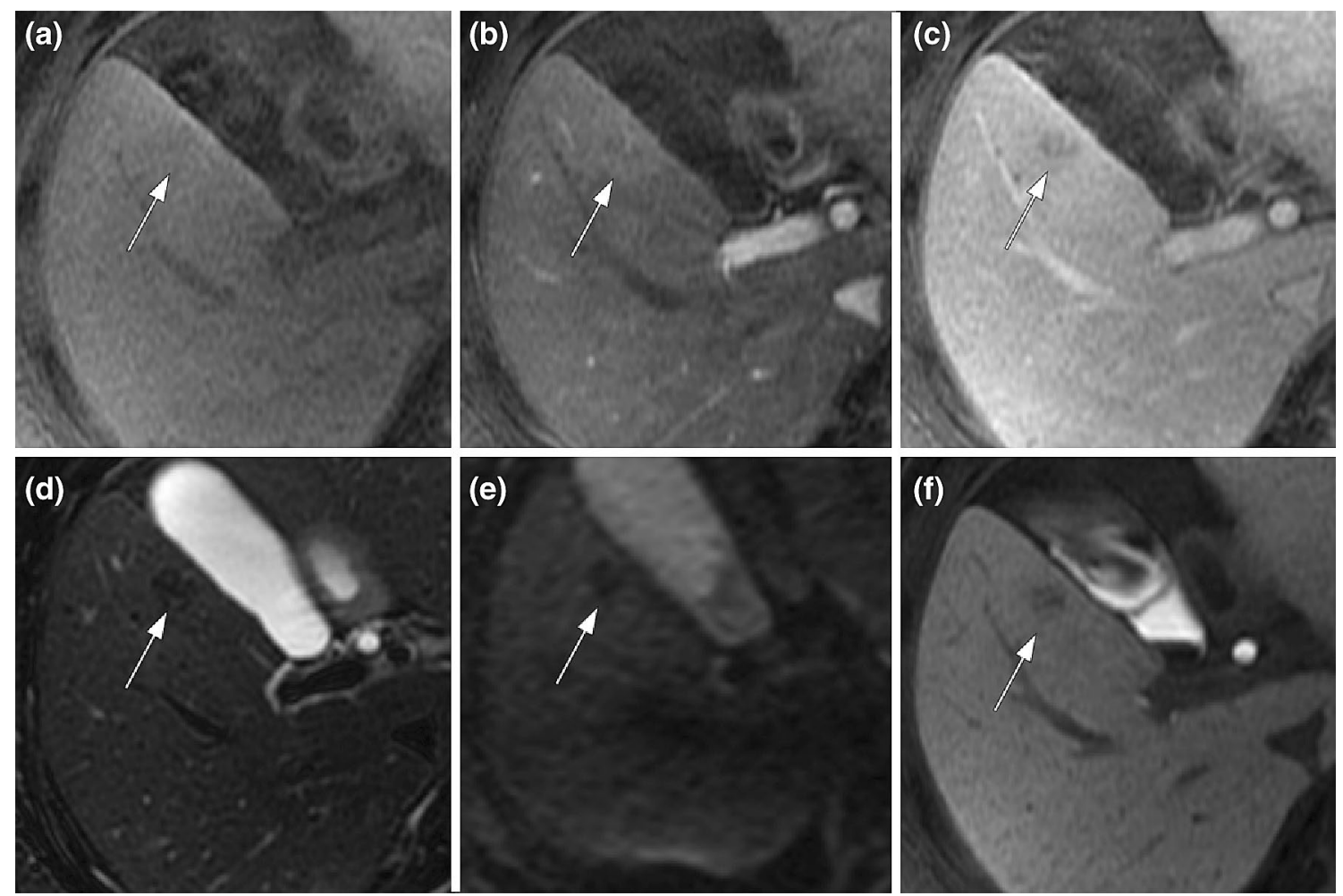

Fig. 1 Early HCC in 55 year old male with liver cirrhosis (HCV). a Fat suppressed T1-weighted MR image shows iso to slightly hyperintense nodule (arrow). b Arterial dominant phase of gadoxetic acidenhanced MRI shows a 1.2-cm-diameter nodule without arterial hyper enhancement (arrow). c Transitional phase of gadoxetic acidenhanced MRI shows slight hypointensity (arrow). d Fat suppressed

within the tumor. A previous study documented portal vein invasion (27\%) and intrahepatic metastasis (10\%) in classic small HCC [21]. Radiologically classic small HCC tends to show a typical enhancement pattern of HCC such as arterial hypervascularity and corona pattern of enhancement [18] (Fig. 2). Therefore, even in small HCCs less than $2 \mathrm{~cm}$, early HCC shows a more favorable biological behavior and prognosis than small classic HCC showing hyperenhancement on arterial dominant phase with distinct margin. On the other hand, poorly differentiated HCC demonstrates an invasive nature with different imaging features as described below even when smaller (Fig. 3).

\section{Multifocality}

The number of tumors is another important prognostic factor [1]. The number of HCCs was reported to correlate positively with microvascular invasion [22]. There are two types of multifocal HCCs, namely, multicentric HCC and intrahepatic metastases from a primary HCC (including satellite nodules).
T2-weighted MR image shows hypointensity of the nodule (arrow). e Diffusion-weighted image shows hypointensity of the nodule (arrow). f Hepatobiliary phase (HB phase) of gadoxetic acid-enhanced MRI shows slight hypointensity of the nodule (arrow). This nodule was confirmed as early HCC pathologically

Intrahepatic metastases from $\mathrm{HCC}$ tend to show a worse prognosis compared with multicentric HCC [23]. Although it is difficult to strictly distinguish between them radiologically, they have the respective characteristics noted below. Multicentric HCC has various histological grades. And a nodule in nodule appearance or well-differentiated HCC should be regarded as multicentric HCC. Intrahepatic metastases (IM) resemble the primary lesion with advanced tumor grade.

\section{Macroscopic morphology/gross classification}

The gross classification of HCC is an important prognostic factor [2]. Eggel's gross classification of HCC (nodular, massive, and diffuse) was introduced in 1901 based on autopsy reports [24]. According to the classification proposed by the Liver Study Group of Japan, Eggel's nodular type is further subclassified into the following five types: small nodular type with indistinct margin (SN-IM), simple nodular type (with distinct margin) (SN-DM), simple 

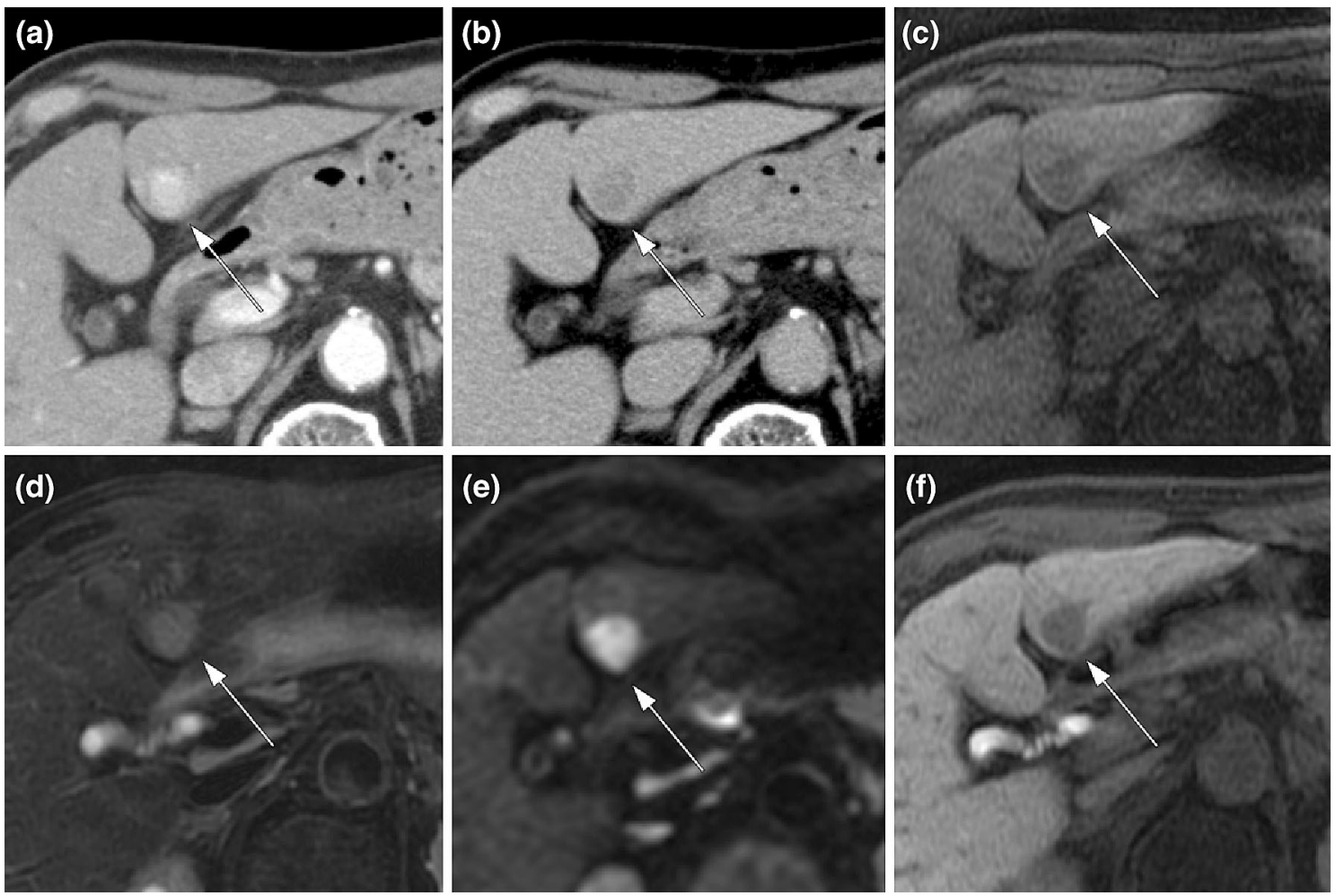

Fig. 2 Classic small HCC in 80 year old male with chronic hepatitis (HCV). a Arterial dominant phase of dynamic CT shows arterial enhancement of nodule measuring $1.6 \mathrm{~cm}$ in diameter (arrow). b Delayed phase of dynamic CT shows hypoattenuating nodule (arrow). c Fat suppressed T1-weighted MR image shows hypointense nodule (arrow). d Fat suppressed T2-weighted MR image shows moderate

nodular type with extranodular growth (SN-EG), confluent multinodular type (CMN) and infiltrative type (Fig. 4) [25]. Of them, the non-simple nodular types (SN-EG, CMN and infiltrative) generally demonstrate a poor prognosis. The microvascular invasion rate of SN-EG, CMN and infiltrative type was reported to be significantly higher than that of SN-DM [2, 26, 27].

Radiologically, the post-vascular phase of sonazoidenhanced ultrasonography has been reported as useful in predicting the macroscopic findings [28]. And the hepatobiliary phase (HB phase) of gadoxetic acid-enhanced MRI could predict the macroscopic pathological findings except for SN-IM [29]. The shape and tumor margins on imaging reflect well the gross classification of HCC.

\section{Macroscopic morphology/fibrous capsule}

A fibrous capsule surrounding $\mathrm{HCC}$ is a favorable prognostic factor [3, 4]. 15-76\% of HCCs have been reported as having a fibrous capsule [30,31]. A fibrous capsule is composed of two layers histologically. The inner layer has a rich fibrous hyperintensity of the nodule (arrow). e Diffusion-weighted image $(b=800)$ shows hyperintensity of the nodule (arrow). f Hepatobiliary phase (HB phase) of gadoxetic acid-enhanced MRI shows the nodule is hypointense (arrow). This nodule was confirmed to be a moderately differentiated HCC pathologically

component and outer layer has various numbers of small vessels and newly formed bile ducts [32,33]. A fibrous capsule is not a common pathological feature of early HCC, dysplastic nodules (DNs), or regenerative nodules [19]. Radiologically, on dynamic CT and MRI an enhancing rim surrounding an HCC on portal venous phase or delayed phase suggests the presence of a fibrous capsule [34]. On MR imaging sequence, the fibrous capsule shows a thin rim of hypointensity on T1WI and hypointensity or hyperintensity on T2WI. Pseudocapsule represents compressed fibrous tissue surrounding regenerative nodules but it has been not well defined [32, 34], and the precise discrimination between capsule and pseudocapsule is often difficult even histologically. The so-called pseudocapsule is thin as compared with fibrous capsule, but both show similar imaging features. HCCs with pseudocapsule formation are also associated with a more favorable prognosis [32, 34]. 

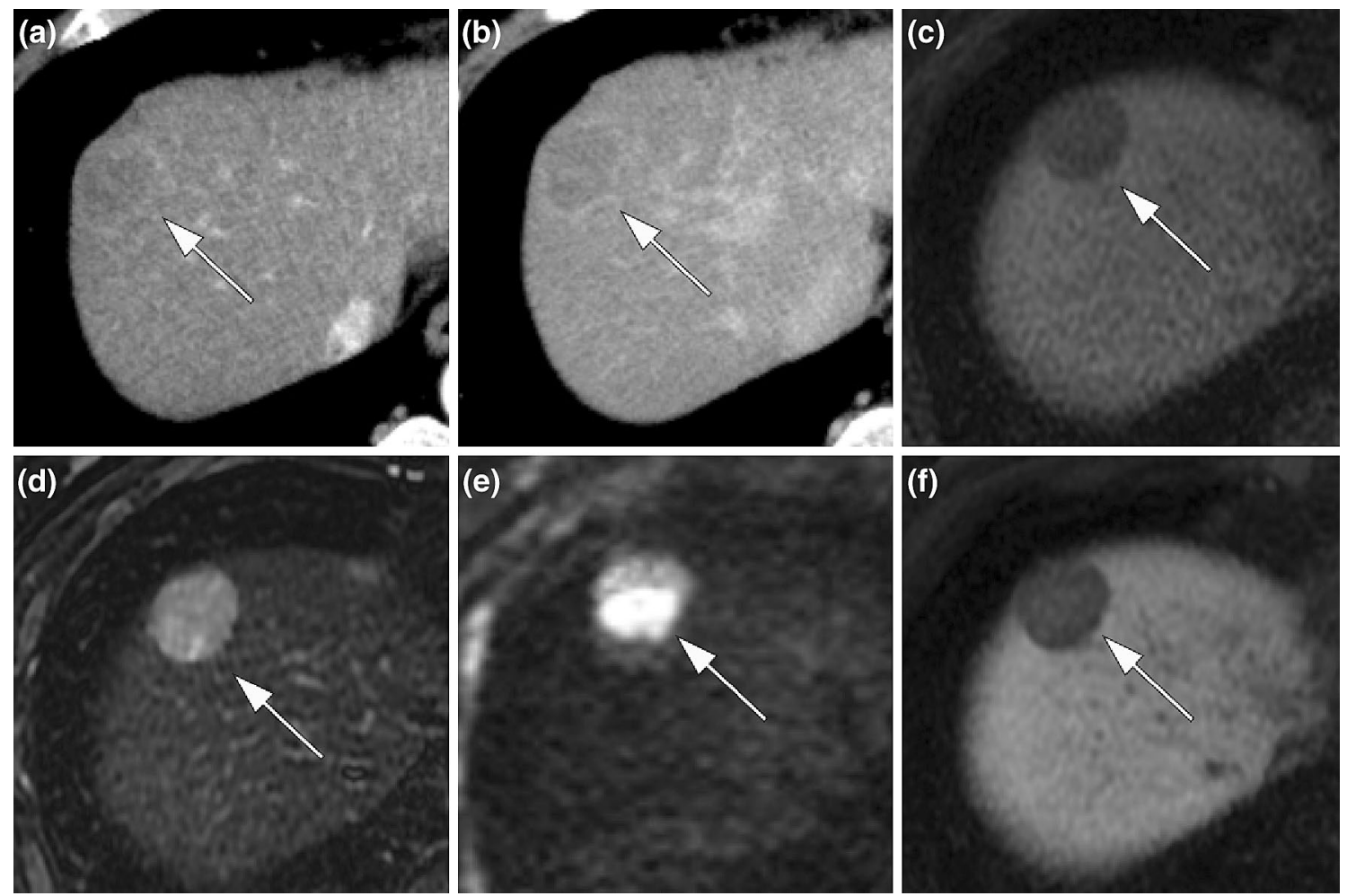

Fig. 3 Poorly differentiated HCC in 56 year old male with chronic hepatitis (HCV). a Arterial dominant phase of dynamic CT shows a 2.4-cm-diameter hypoattenuating nodule compared with the surrounding liver (arrow). b Delayed phase of dynamic CT shows washout of the nodule (arrow). c Fat suppressed T1-weighted MR image shows hypointense nodule (arrow). d Fat suppressed T2-weighted

\section{Grade of differentiation of HCC}

The histological grade of differentiation in $\mathrm{HCC}$ cells is one of the important prognostic factors determining recurrence and survival rates after surgical resection and liver transplantation $[5,6]$. The grade of differentiation in HCC can be predicted by the following imaging biomarkers: (1) intratumoral blood supply, (2) gadoxetic acid uptake, (3) Kupffer cell imaging, (4) signal intensity on T1, T2-weighted imaging and the value of apparent diffusion coefficient (ADC) on diffusion weighted imaging (DWI), and (5) accumulation of fluorodeoxyglucose (FDG) uptake.

\section{Intra-tumoral blood supply}

Estimation of the intra-nodular arterial and portal blood supply is useful to predict the grade of differentiation of HCC. In the course of hepatocarcinogenesis, first both the portal blood supply and hepatic arterial supply decrease (due to a decrease in the number of portal tracts) in parallel with increasing grade of malignancy, after which the number of newly formed abnormal arteries increases. In moderately
MR image shows hyperintense nodule (arrow). e Diffusion-weighted image $(b=800)$ shows hyperintensity of the nodule (arrow). f Hepatobiliary phase (HB phase) of gadoxetic acid-enhanced MRI shows definite hypointense nodule (arrow). This nodule was confirmed as poorly differentiated HCC pathologically

differentiated HCC (classic HCC), the portal blood supply vanishes with only abnormal arteries supplying the lesion $[35,36]$. In the late stage of HCC development (poorly differentiated HCC), arterial vascularity decreases again [37], probably due to increased anaerobic metabolism, and it is often shown as a hypovascular tumor compared with background liver (Fig. 3).

\section{Gadoxetic acid uptake}

The expression of organic anion-transporting polypeptide (OATP)1B3 (thought to be main uptake transporter of gadoxetic acid in HCC cells) is significantly decreased in accordance with increasing grade of malignancy of the nodules (Fig. 5), and around $80 \%$ of early HCCs already demonstrate decreased but not absent OATP1B3 expression relative to the surrounding liver parenchyma, resulting in slight hypointensity on the hepatobiliary phase of gadoxetic acid MRI (Fig. 1). All poorly differentiated HCCs show absent or markedly decreased expression with definite hypointensity on HB phase (Fig. 3). Well and moderately differentiated HCCs demonstrate 


\begin{tabular}{|l|c|c|c|c|}
\hline & $\begin{array}{c}\text { Small nodular } \\
\text { type with } \\
\text { indistinct } \\
\text { margin (SN-IM) }\end{array}$ & $\begin{array}{c}\text { Simple nodular } \\
\text { type with distinct } \\
\text { margin } \\
\text { (SN-DM) }\end{array}$ & $\begin{array}{c}\text { Simple nodular } \\
\text { type with } \\
\text { extranodular } \\
\text { growth (SN-EG) }\end{array}$ & $\begin{array}{c}\text { Confluent } \\
\text { multinodular } \\
\text { type (CMN) }\end{array}$ \\
\hline Schema & $\ldots$ & & & \\
\hline $\begin{array}{l}\text { Macroscopic } \\
\text { appearance }\end{array}$ & & & &
\end{tabular}

Fig. 4 Gross classification of HCC. Eggel's nodular type HCC is subclassified into five types: small nodular type with indistinct margin (SN-IM), simple nodular type with distinct margin (SN-DM), simple nodular type with extranodular growth (SN-EG), confluent multinodular type (CMN) and infiltrative type. Among them, common types, small nodular type with indistinct margin (SN-IM), simple nodular type with distinct margin (SN-DM) and confluent multinodular type (CMN) schemas, macroscopic appearance, and HB phase finding of gadoxetic acid enhanced MRI (EOB-MRI) are shown. Modified from reference [25]

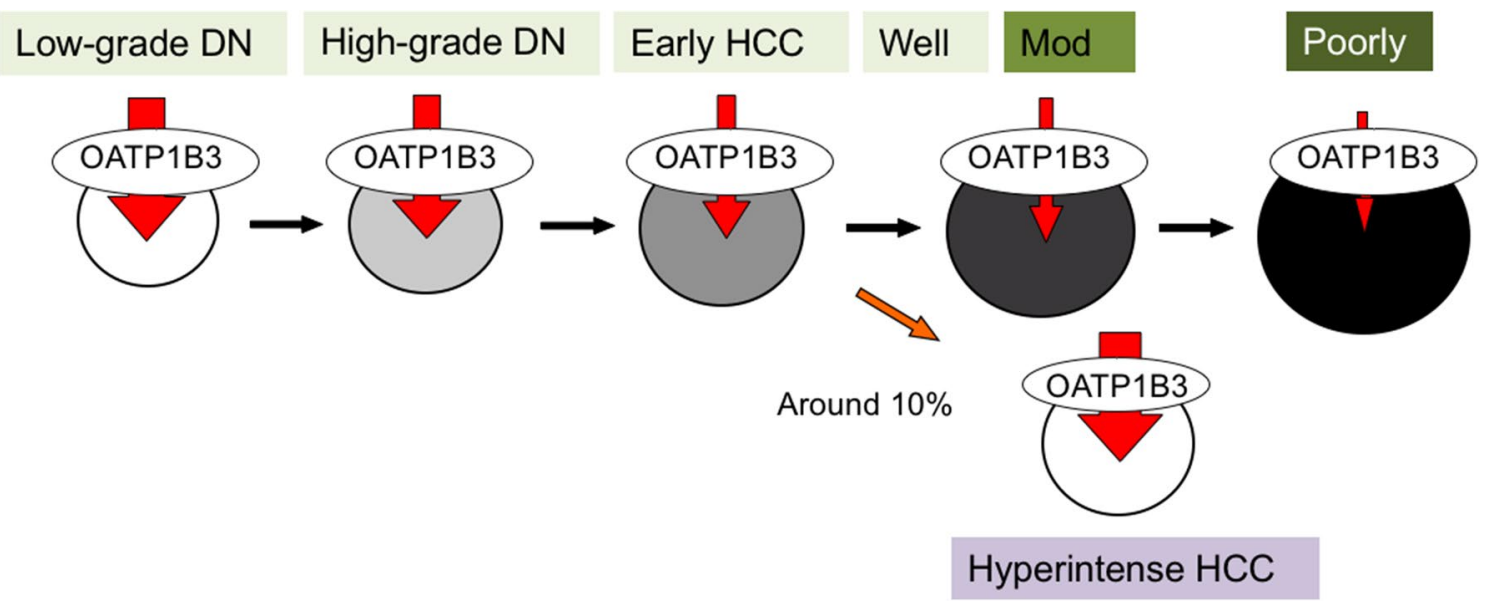

Fig. 5 Grade of gadoxetic acid uptake in HB phase is useful for predicting the grade of differentiation of HCC. The expression of OATP1B3 (main uptake transporter of gadoxetic acid in HCC) is significantly decreased in parallel with increasing grade of malignancy of the nodules. Around $80 \%$ of early HCCs already demonstrate decreased but not absent OATP1B3 expression relative to the surrounding liver parenchyma. All of the poorly differentiated HCCs show absent or markedly decreased expression. Well and moderately differentiated HCCs demonstrate an intermediate grade of OATP1B3 expression between early HCC and poorly differentiated HCCs. Around $10 \%$ of them show equivalent or increased expression relative to the surrounding liver. Signal intensity of HB phase is useful for predicting the grade of differentiation of HCC. Modified from reference [38] 
an intermediate grade of OATP1B3 expression between early HCC and poorly differentiated HCCs (Fig. 2), but around $10 \%$ of them show equivalent or increased expression relative to the surrounding liver [38] (Fig. 5). Signal intensity of $\mathrm{HB}$ phase is useful for predicting the grade of differentiation of HCC (Figs. 1, 2, 3).

\section{Kupffer cell imaging (SPIO-MRI or Sonazoid US)}

Superparamagnetic iron oxide (SPIO)-MRI and gaseous perfluorobutane (Sonazoid, GE Healthcare) can be used for Kupffer cell imaging. Kupffer cell imaging is useful for estimation of the histological grade of HCCs, although there is some difficulty in the differentiation between dysplastic nodules and well-differentiated HCCs. The number of Kupffer cells decreases in parallel with increasing grade of differentiation of HCCs and most well-differentiated HCCs show a similar number of Kupffer cells in the tumors to that in surrounding non-tumor tissues [39]. Signal intensity of HCC relative to the surrounding liver on SPIO enhanced T2WI increases as the degree of differentiation of HCCs declines. However, considerable overlap has been noted between the SPIO intensity of dysplastic nodules and that of well-differentiated HCCs [39]. Contrast-enhanced ultrasound (US) using Sonazoid is also a useful tool for estimating the histologic grade of HCC [40]. The proportion of hypoechoic tumors during the Kupffer phase is increased from well-differentiated to moderately and poorly differentiated HCCs.

\section{Signal intensity on T1WI and T2WI and the value of ADC}

About $65 \%$ of well-differentiated HCC show hyperintensity on T1WI [41]. Hyperintensity on T1WI of HCC gradually decreases in parallel with increasing histological grade [41]. Therefore, HCCs with T1WI hyperintensity tend to have a better tumor histologic grade, while HCCs with T1WI hypointensity tend to be more poorly differentiated [41, 42]. In addition, T1WI hyperintense HCCs without T2WI hyperintensity or arterial hypervascularity usually show a benign clinical course [43].

Higher signal intensities on T2WI have been related to a lower degree of histologic differentiation in HCC [33, 42] (Figs. 1, 2, 3).

Quantitative measurement of ADC on DWI of HCC is a predictor of histological grade $[44,45]$ and early recurrence before treatment [44]. ADC value of HCC significantly decreases in parallel with increasing histological grade. Poorly differentiated HCC shows lower ADC values compared with all other histological grades.

\section{Fluorodeoxyglucose (FDG) uptake}

${ }^{18}$ F-fluorodeoxyglucose positron emission tomography (FDG-PET) has a low sensitivity for detecting well to moderately differentiated HCCs [46], because of lower expression of HK (hexokinase) and GLUT1 (glucose transporter 1) and higher expression of G6Pase (glucose-6-phosphatase) in well to moderately differentiated HCCs. High FDG accumulation in poorly differentiated HCC indicates increased GLUT1 and decreased G6Pase [46]. FDG accumulates similarly in highly differentiated HCC and normal liver, with the signal strength of FDG being relatively weak, making FDG uptake a predictor of the grade of HCC differentiation. Therefore, FDG uptake can serve as one of the predictors of the grade of HCC differentiation.

In summary, the grade of differentiation of HCC during multistep hepatocarcinogenesis can be well predicted by the combination of the imaging biomarkers including intratumoral blood flow, signal intensity of HB phase, SPIO uptake, signal intensity of T1WI and T2WI, ADC value of DWI, and FDG uptake (Fig. 6).

\section{Macrovascular invasion}

There are two types of vascular invasion, macro and microvascular invasion, depending on the level of involved vascular structures. Both macro and microvascular invasion are associated with a poor prognosis because they provide the route for tumor cells to access the portal or systemic circulation. HCCs with vascular invasion have frequent intrahepatic metastasis and a higher recurrence rate after hepatic resection, ablation therapy, or liver transplantation [7, 8]. Therefore, surgical resection or liver transplantation is usually contraindicated in HCCs with macrovascular invasion [47].

Vascular invasion is more common in HCCs that are larger or show a higher histologic grade [48, 49]. HCCs more frequently involve the portal venous system than hepatic veins [49].

\section{Microvascular invasion}

The presence of microvascular invasion has been reported to be one of the most important risk factors related to postsurgery tumor recurrence [9]. Despite its significance in HCC assessment, the diagnosis of microvascular invasion is usually made by the postoperative pathological diagnosis. However, the presence of microvascular invasion may be predicted by the following imaging biomarkers: (1) tumor margin (non-smooth margin), (2) peritumoral enhancement (irregular circumferential), (3) peritumoral hypointensity on HB phase of gadoxetic acid-enhanced MRI, (4) ADC, (5) 


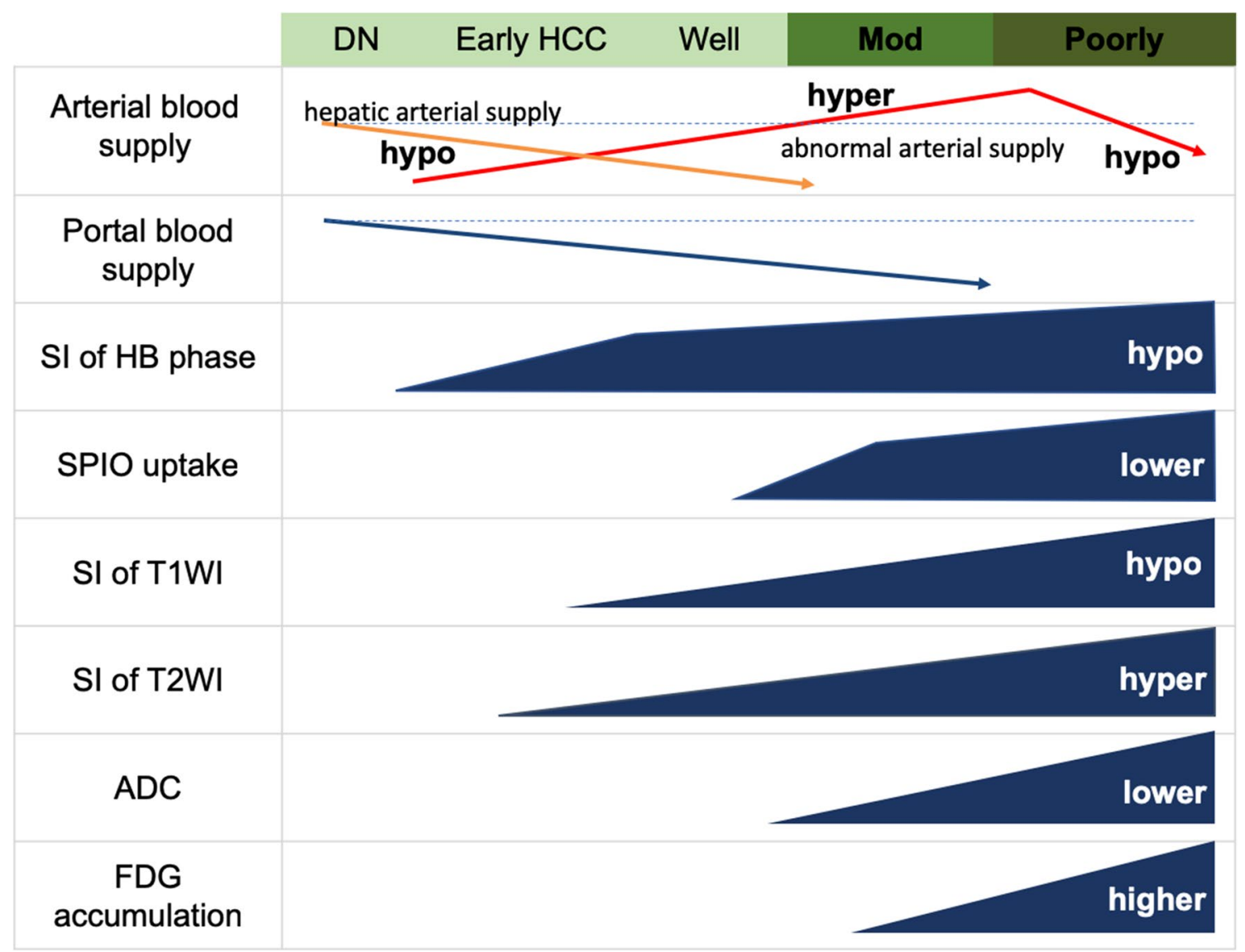

Fig. 6 Summary of the imaging biomarkers predicting the grade of differentiation in HCC. During hepatocarcinogenesis, the frequency of neovascularized arteries increases from dysplastic nodule (DN) to moderately differentiated HCC (Mod). However, arterial vascularity decreases again in poorly differentiated HCC (Por). In parallel with increasing grade of malignancy, the signal intensity of the hepato-

tumor size, (6) arterial rim enhancement, (7) FDG uptake, (8) disruption of the capsule and (9) multifocality.

\section{Tumor margin (non-smooth margin)}

Non-smooth tumor margins (simple nodular type with extranodular growth, and confluent multinodular type), detected on multiphasic CT, have been found to correlate with the pathologic presence and location of microvascular invasion [50]. Ariizumi et al. [51] reported that non-smooth tumor margin on the HB phase of gadoxetic acid-enhanced MRI can be used as a preoperative predictor of microvascular invasion (Fig. 7a).

\section{Peritumoral enhancement (irregular circumference)}

Kim et al. [52] categorized the pattern of peritumoral enhancement as wedge-shaped and irregular circumferential enhancement. The wedge-shaped enhancement was not biliary phase (HB phase) of gadoxetic acid-enhanced MR image and T1-weighted image and SPIO uptake are decreased, while the signal intensity of T2WI is increased. And lower value of apparent diffusion coefficient (ADC) on diffusion-weighted image (DWI) and higher accumulation of FDG are observed in the worse histological grades of HCC

a statistically significant risk factor for microvascular invasion, while irregular circumferential peritumoral enhancement could be a preoperative predictor of microvascular invasion (Fig. 7b). The sensitivity, specificity, positive predictive value (PPV), and negative predictive value (NPV) of irregular circumferential peritumoral enhancement for prediction of microvascular invasion were reported to be $74.3,82.9,81.3$ and $76.3 \%$, respectively. Nishie et al. [53] reported that the area of peritumoral hemodynamic change might be larger in the microvascular invasion group, particularly in small HCCs $(\leq 3 \mathrm{~cm})$.

\section{Peritumoral hypointensity on the HB phase}

Peritumoral hypointensity on the HB phase of gadoxetic acid-enhanced-MRI may also be a predictive factor of microvascular invasion (Fig. 7c). Kim et al. observed peritumoral hypointensity on HB phase in 26 (25.0\%) of 104 HCCs, with $23(88.5 \%)$ of them showing microvascular 

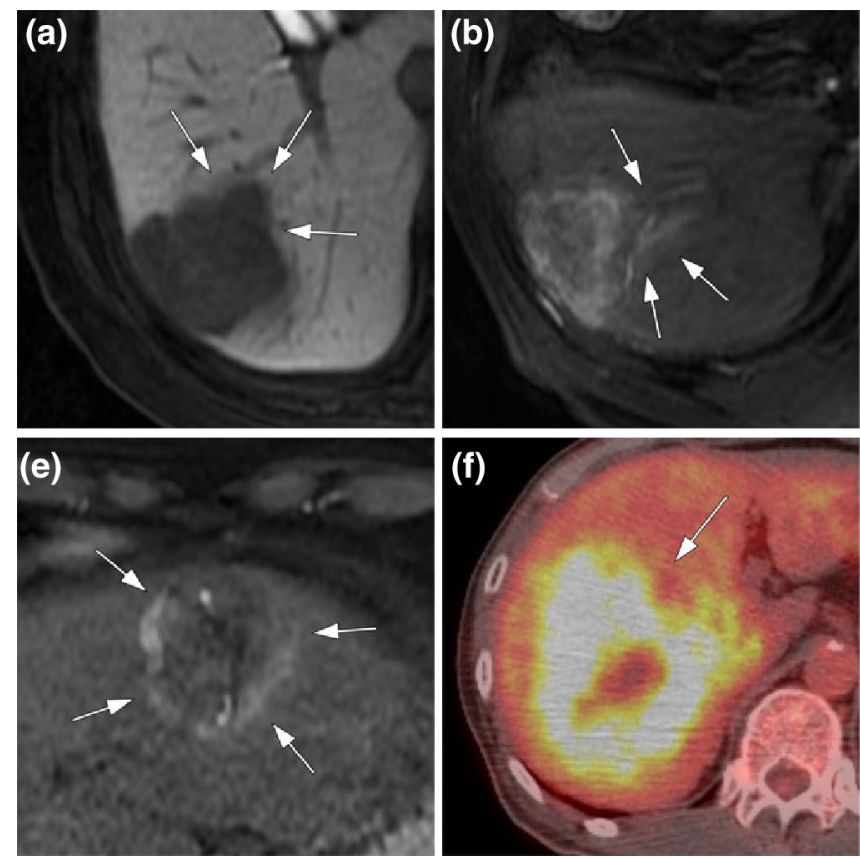

Fig. 7 Imaging biomarkers predicting the presence or absence of microvascular invasion of HCC. a Non-smooth tumor margin on hepatobiliary phase (HB phase) of gadoxetic acid-enhanced MR image. HB phase of gadoxetic acid-enhanced MR image shows a non-smooth tumor margin (arrows). This nodule was confirmed to be a moderately differentiated HCC demonstrating the macroscopic appearance of simple nodular type with extranodular growth (SNEG) with microvascular invasion pathologically. b Irregular circumferential enhancement on arterial dominant phase. This nodule has irregular circumferential enhancement (arrows) on arterial dominant phase of gadoxetic acid-enhanced MRI. This nodule was confirmed to be a poorly differentiated HCC, demonstrating the macroscopic appearance of a simple nodular type with extranodular growth (SNEG) macroscopic appearance with micro portal venous and hepatic venous invasion. The region of peritumoral enhancement presents an irregular crescent or polygonal shape. c Peri-tumoral hypointensity on HB phase of gadoxetic acid-enhanced MR image. HB phase of gadoxetic acid-enhanced MR image shows a hypointense area around the main tumor (arrows). Microvascular invasion was observed path-

invasion. The sensitivity, specificity, PPV, and NPV for predicting microvascular invasion by the presence of peritumoral hypointensity were reported as $38.3,93.2$, 88.5 and $53.6 \%$, respectively. The probable cause of peritumoral hypointensity was considered to be decreased expression of OATPs because of hemodynamic alterations related to tumor obstruction of minute portal veins [54]. Nishie et al. reported similar finding as peritumoral decreased uptake area of gadolinium ethoxybenzyl diethylenetriamine pentaacetic acid (Gd-EOB-DTPA) (POUAE). Based on this study, the presence of PDUAE can be an indicator of microscopic vascular invasion with a sensitivity, specificity, accuracy, positive predictive value, and negative predictive value of $72,80.6,77,72$ and $80.6 \%$, respectively [55].
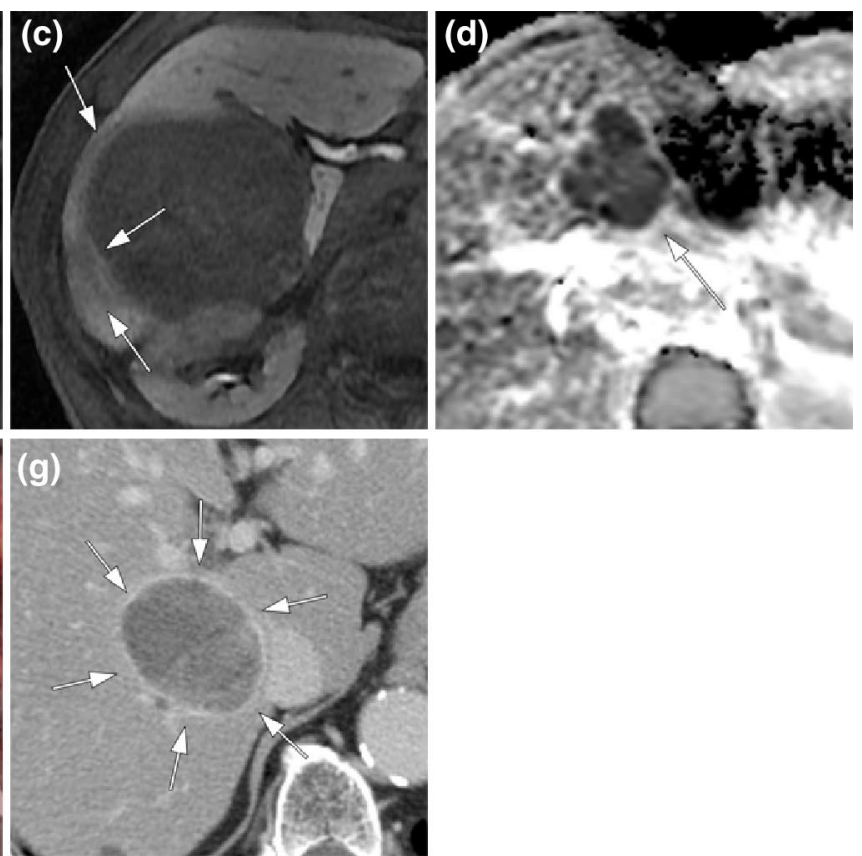

ologically. d Lower ADC value. This moderately to poorly differentiated HCC had a low ADC value (arrow) $\left(1.05 \times 10^{-3} \mathrm{~mm}^{2} / \mathrm{sec}\right)$ and microvascular invasion pathologically. e HCC with arterial rim enhancement. Arterial dominant phase of gadoxetic acid-enhanced MRI shows HCC with arterial rim enhancement (arrows). This HCC was confirmed to be a poorly differentiated HCC demonstrating the macroscopic appearance of a simple nodular type with extranodular growth (SN-EG) with micro portal venous invasion pathologically. f High accumulation HCC on FDG-PET (arrow). This poorly differentiated HCC demonstrating the macroscopic appearance of a simple nodular type with extranodular growth (SN-EG) shows high accumulation of FDG (SUV max 9.6), with micro hepatic venous invasion confirmed pathologically. g Intact HCC capsule on delayed phase of dynamic CT. Delayed phase of dynamic CT shows intact HCC capsule (arrows). This HCC was confirmed to be a well-differentiated HCC demonstrating the macroscopic appearance of a simple nodular type with distinct margin (SN-DM) without microvascular invasion pathologically

\section{ADC value}

Suh et al. [56] showed that lower ADC values can be a useful predictor of microvascular invasion (Fig. $7 \mathrm{~d}$ ), with a sensitivity and specificity of $93.5 \%$ and $72.2 \%$, respectively, and a cut off ADC value of $1.11 \times 10^{-3} \mathrm{~mm}^{2} /$ second.

\section{Tumor size}

Tumor size shows a statistically significant association with the presence of microvascular invasion [57, 58]. Significant tumor size cutoffs for positive microvascular invasion were reported as $>5 \mathrm{~cm}(P=0.001)$ by Ahn et al. [57], and $\geq 3 \mathrm{~cm}$ $(P=0.0013)$ by Hirokawa et al. [58]. 


\section{Arterial rim enhancement}

An et al. [59] reported that arterial rim enhancement on arterial phase of dynamic MRI is associated with microvascular invasion (Fig. 7e). The arterial rim enhancement pattern in $\mathrm{HCC}$ has also been reported to indicate rapid progression and poorer differentiation $[60,61]$.

\section{FDG-PET uptake}

Kornberg et al. [62] reported that preoperative ${ }^{18} \mathrm{~F}$-FDG uptake on PET was a reliable predictor of microvascular invasion (Fig. 7f). Ahn et al. [57] reported that a ratio of tumor maximum standardized uptake values (SUV) to normal liver mean SUV (TSUVmax/L SUVmean) of 1.2 or more had a statistically significant association with microvascular invasion $(P<0.001)$.

\section{Disruption of the capsule}

Lim et al. [30] reported that the disruption of the capsule on CT to be correlated with microvascular invasion $(P<0.001)$, and the presence of an intact HCC capsule on CT was closely correlated with the absence of microvascular invasion (Fig. 7g). However, in a different study, no significant correlation was noted between microvascular invasion and the presence of a capsule [52]. The correlation between presence of a capsule and microvascular invasion thus remains unclear.

\section{Multifocality}

Chandarana et al. reported that the presence of three or more tumors on MRI and four or more at pathologic examination had high specificity ( $88.2 \%$ and $91.2 \%$, respectively) for the prediction of microvascular invasion [22]. However, a recent study documented that multifocality was not associated with microvascular invasion [57]. These discordant results may be attributable to differences between intrahepatic metastasis and multicentric hepatocarcinogenesis. In this way, tumor multifocality remains a controversial parameter for prediction of microvascular invasion in HCC.

As mentioned above, imaging biomarkers predicting the presence or absence of microvascular invasion in HCC are non-smooth tumor margin, irregular circumferential peritumoral enhancement, lower ADC value, increased tumor size, arterial rim enhancement and increased FDG uptake, capsule disruption and multifocal tumor. Microvascular invasion can be predicted to some extent by these imaging findings. Interestingly, a recent study revealed that the combination of two or more MR imaging biomarkers such as arterial peritumoral enhancement, non-smooth tumor margin, and peritumoral hypointensity on HBP can be used as a preoperative imaging biomarker for predicting microvascular invasion with a high specificity (90\%) and an association with early recurrence after curative resection of single HCC [63].

\section{Bile duct invasion}

Around 1.2-9\% of HCCs show bile duct invasion [64]. HCC patients with bile duct invasion are thought to have a poorer prognosis than those without it [10]. The reasons for the poor prognosis of HCC patients with bile duct invasion include the following. HCC with bile duct invasion is often accompanied by obstructive jaundice, cholangitis and hemobilia that are also immediate threats to survival, HCC with bile duct invasion is frequently accompanied by portal vein invasion, while HCC with bile duct invasion has been shown to have a more advanced tumor stage $\mathrm{HCC}$ with adverse histological features including higher rates of microvascular invasion, lymphovascular invasion and poor differentiation [65]. The long-term outcomes after surgical treatment for HCC with bile duct invasion are still controversial. Some previous studies reported poorer surgical outcomes in these patients than in those without bile duct invasion [66]. However, others have reported that some patients with obstructive jaundice can be treated by hepatic resection, with favorable long-term results [67].

\section{Intra-tumoral fat}

The presence of intra-tumoral fat has been documented in $19.6 \%$ of HCCs on light microscopy [68] or up to $10 \%$ of HCCs on opposed-phase of MRI [33]. Patients with fatcontaining HCC may have a better clinical outcome than patients without it [11]. Diffuse fatty metamorphosis is considered to be one of the characteristics of early-stage HCC and well-differentiated HCC [68]. Approximately 6\% of moderately differentiated HCCs have fatty change, in contrast to poorly differentiated $\mathrm{HCCs}$ in which it is rarely seen [68]. On the other hand, Asayama et al. reported that a fat component was significantly more frequent in poorly differentiated HCC compared to the moderately differentiated $\mathrm{HCC}$ and proposed a possible mechanism of fatty change in relation to decreased arterial blood supply [69]. There is a controversial point regarding the amount of fat component in poorly differentiated HCC. Min et al. [70] reported lower rates of microvascular invasion in fat-containing HCCs (27.3\%), than non-fat-containing HCCs (39.1\%), although this difference was not statistically significant $(P=0.07)$. In addition, diffuse-fat-containing HCCs showed a lower tendency for microvascular invasion $(21.1 \%)$ compared to that of focal-fat-containing HCCs (35.7\%) or non-fat-containing HCCs (36.8\%). HCC with intra-tumoral fat may have a more 
favorable prognosis, but the clinical value of this finding remains uncertain.

\section{Molecular factors}

\section{FDG PET}

A meta-analysis showed both high Tsuv/Lsuv ratio and high Tsuv value to be associated with a poor prognosis in HCC patients. Therefore, pretreatment ${ }^{18} \mathrm{~F}$-FDG PET is a useful tool in predicting the prognosis of HCC patients [12].

\section{HB phase hyperintense HCC}

Around $10 \%$ of hypervascular classic HCCs [71, 72] show hyperintensity (HB phase hyperintense HCC) on HB phase of gadoxetic acid-enhanced MRI (Fig. 8). Compared with hypervascular HCC showing hypointensity on HB phase, HB phase hyperintense HCC (OATP1B3 overexpressed HCC) shows a biologically less aggressive nature with a good prognosis, higher frequency of well to moderate differentiation and rarer portal vein invasion [13], lower expression of $\alpha$-fetoprotein (AFP), AFP L3 and protein induced by vitamin K absence or antagonists-II (PIVKA-II) [13], lower recurrence rate and better prognosis [13], beta-catenin, glutamine synthetase (which is a target of Wnt/ $\beta$-catenin signaling), Hep-Par1 (hepatocyte marker) and hepatocyte nuclear factor $4 \alpha(\mathrm{HNF} 4 \alpha)$ by immunohistochemical analysis [73, 74] and weaker expression of stem cell markers [epithelial cell adhesion molecule (EpCAM), cytokeratin 19 (CK19)] by immunohistochemical analysis [75].

Hyperintense $\mathrm{HCC}$ is a molecular/genetical subtype of HCC with activation of mature hepatocyte-related genes/ pathways and hepatocyte nuclear factor $4 \alpha$ overexpression
[75]. HNF4 $\alpha$ suppresses hepatocyte proliferation and experimental HCC growth, and its decrease is linked to hepatocarcinogenesis [76]. Of note, a recent study reported that the inhibitory effect of $\mathrm{HNF} 4 \alpha$ on $\mathrm{HCC}$ development might be caused by suppression of hepatocyte epithelial-mesenchymal transformation (EMT) and cancer stem cell generation [76].

\section{Genome-based molecular classification of HCC}

In the past decade, molecular-based HCC classification has been elucidated [14, 15]. Recently, Calderano et al. [16] also proposed six subclasses (G1-G6) of HCC molecularbased classification associated with clinical and histological features (Fig. 9). Groups G1-G3 are characterized by high cell proliferation and chromosomal instability with enrichment in TP53 (G1-3), RPS6KA3 (G1), AXIN1 and ATM (G1-2), and FGF19 and TSC1/TSC2 (G3) genetic alterations. And G1-G3 have the following clinical features: HBV infection (G1-2), high AFP serum levels (G1-3), female gender (G1) and haemochromatosis (G3). Histologically, G1-3 HCCs were poorly differentiated (G1-3) with frequent macrovascular invasion (G3) foci of clear cells (G1), sarcomatous changes (G1G2), areas of compact (G3) and macrotrabecular (G3) histological patterns. The G1 subtype showed a progenitor phenotype with both CK19 and EpCAM expression. On the other hand, groups G4-G6 are characterized by chromosomal stability. G5G6 subclasses strongly associated with catenin beta $1(C T N N B 1)$ mutations (G5G6) and G4 HCCs have the genetic feature without $C T N N B 1$ and TP53 mutations. G4-G6 HCCs have the following histological features: good differentiation (G4-6), steatohepatitic subtype (G4),
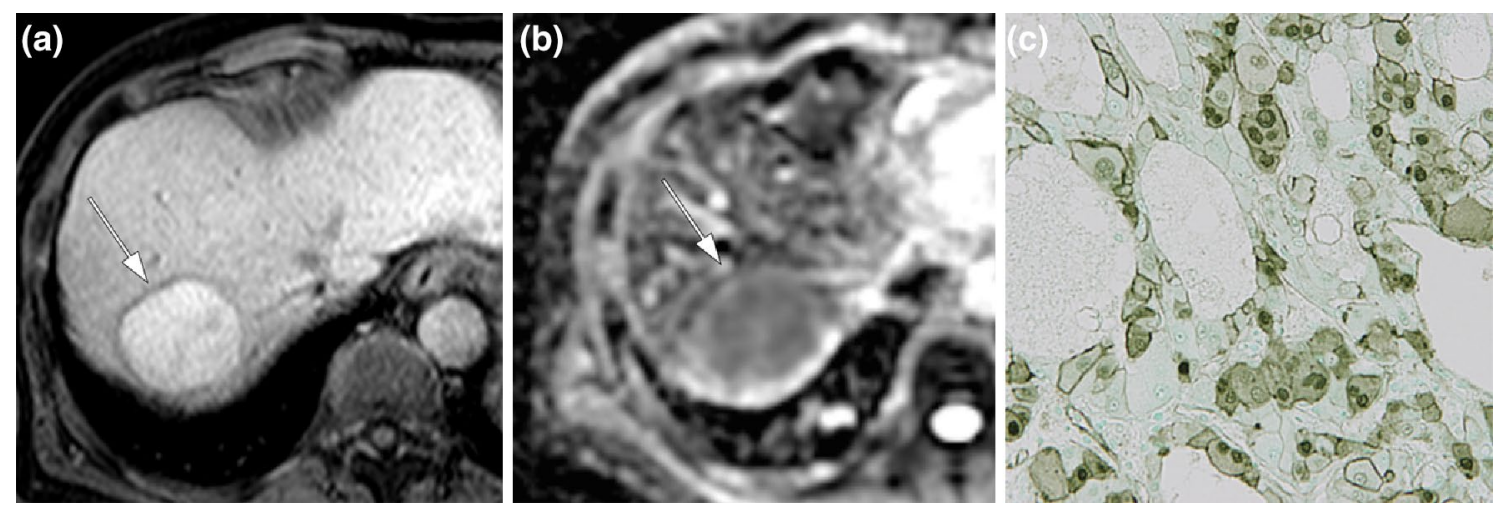

Fig. 8 Hyperintense $\mathrm{HCC}$ on hepatobiliary phase (HB phase) of gadoxetic acid-enhanced MR confirmed as CTNNB-1 mutated HCC in 66 year old male with liver cirrhosis (alcoholic). a Hepatobiliary phase (HB phase) of gadoxetic acid-enhanced MR image shows hyperintense nodule (arrow). b Apparent diffusion coefficient (ADC) map shows relatively high $\left(1.42 \times 10^{-3} \mathrm{~mm}^{2} / \mathrm{sec}\right)$ value in the nodule (arrow). c Pathological diagnosis is moderately differentiated HCC with pseudoglandular pattern. Immunohistochemical staining of the resected specimen for beta-catenin shows nucleus beta-catenin staining of the tumor cells. (original magnification $\times 200$ ) 


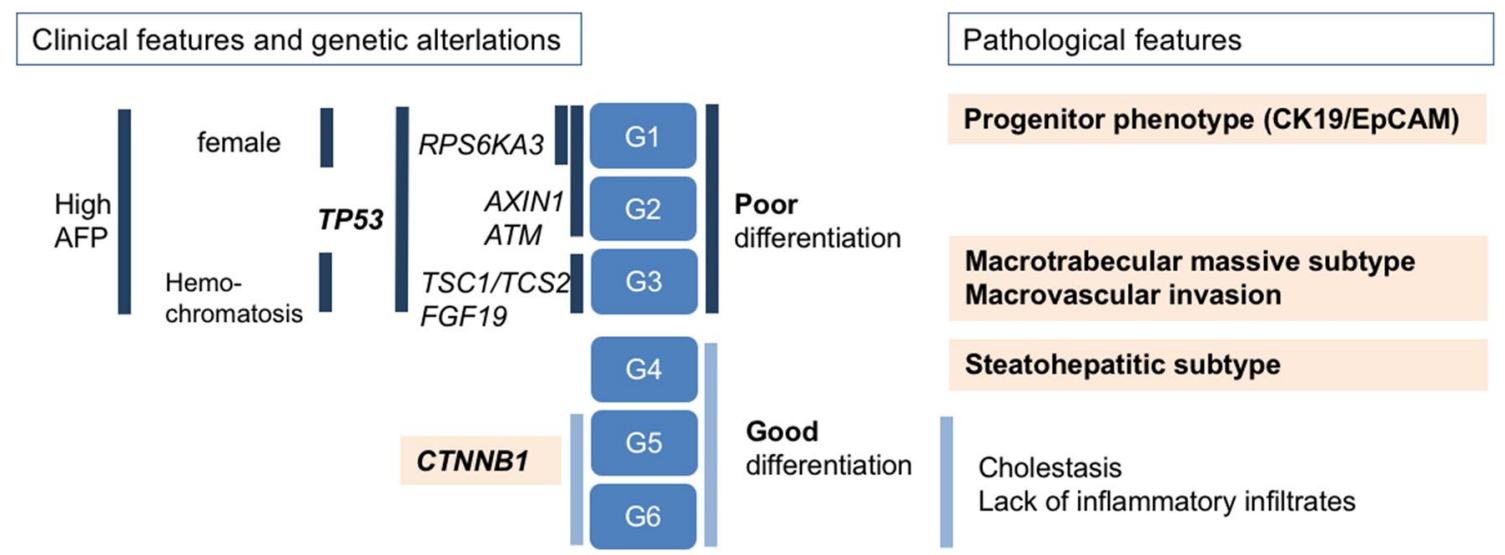

Fig. 9 Genome-based molecular classification of HCC. Recent study proposed six subclasses (G1-G6) of HCC molecular based classification associated with the clinical and histological features. Modified from reference [16]

microtrabecular pattern of growth (G5G6), the presence of tumor cholestasis and a lack of inflammatory infiltrates (G5G6).

\section{CK19 positive HCC}

CK19 has been considered as a marker for the biliary phenotype and stem or progenitor cell. CK19 positive HCC has TP53 inactivation mutations. CK19 positive HCC has been shown to have a high metastatic potential, which is associated with a poor prognosis [77].

Radiologically hypovascular HCCs have been significantly associated with positive CK19 expression [78] (Fig. 10). A recent study revealed that on gadoxetic acidenhanced MRI irregular margin, arterial rim enhancement, lower tumor-to liver ADC ratio, and lower tumor to liver SI ration at HB phase may be useful to predict CK19positive HCC with early recurrence after surgery [79].

\section{EpCAM positive HCC}

EpCAM positive HCC shows an aggressive biological behavior and poor prognosis with stem cell features. It is established that EpCAM is a biomarker for normal hepatic stem cells and cancer stem cells (CSC) in HCC [80]. EpCAM positive HCC associated with worse histological grade and high serum AFP level [81], and displayed a distinct molecular signature with features of hepatic stem cell/progenitor markers such as CK19 and c-kit and activated with Wnt-beta-catenin signaling [82]. Therefore, CK19 positive HCC and EPCAM positive HCC seem to overlap to some extent. The imaging features of EpCAM positive $\mathrm{HCC}$ are still unclear.

\section{Macrotrabecular massive HCC (MTM-HCC)}

MTM-HCC has clinical and biological relevance. MTM$\mathrm{HCC}$ has poor survival, and $10 \%$ of HCCs are classified as MTM-HCC subtype [16]. MTM-HCC is associated with TP53 inactivation, ATM mutations, HBV infection, angiogenesis activation, high AFP serum level, satellite nodules and macro and microvascular invasion with early relapse and poor survival, and frequently demonstrated progenitor phenotype. Thick trabeculae surrounded by vascular spaces $(>50 \%)$ are a characteristic pathological feature. The imaging features of MTM-HCC have not yet been described.

\section{Steatohepatitic HCC (SH-HCC)}

13.5-35.5\% of HCCs have been reported to be of the steatohepatitic subtype [83]. This subset of HCCs is associated with metabolic conditions and the presence of steatosis or steatohepatitis in the background liver. Lack of WNT/betacatenin pathway activation is one of the genomic features. Pathological features of SH-HCC include large droplet steatosis, ballooning of malignant hepatocytes, Mallory-Denk bodies, pericellular fibrosis and intra-tumoral inflammatory cell infiltration.

The biological nature of SH-HCC is still controversial. Goossens et al.[15] reported SH-HCC as being associated with $\mathrm{S} 1$ subclass with more aggressive tumor behavior. On the other hand, Calderaro et al. [16] pointed out that SH$\mathrm{HCC}$ is not related to specific clinical features, but displays a less aggressive phenotype with a lack of satellite nodules and microvascular invasion. Further investigation regarding this issue is needed. One of the characteristic imaging features of SH-HCC is fat deposition, although the details of the imaging features of SH-HCC remain to be clarified (Fig. 11). 

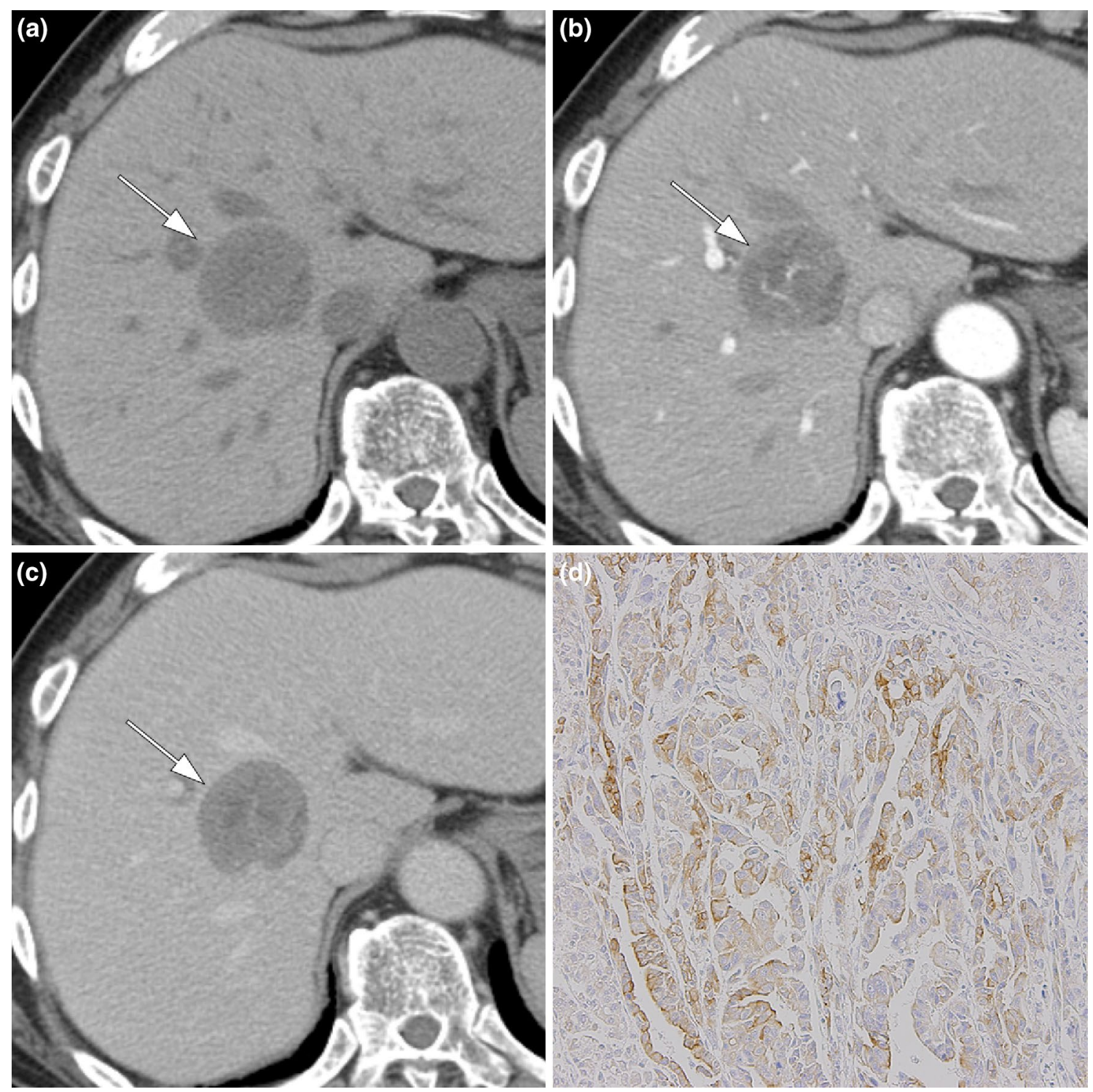

Fig. 10 CK19 positive HCC in 75 year old male with liver cirrhosis (non B, C). a Pre-contrast CT shows hypoattenuating nodule (arrow) on segment 8/1. b Arterial dominant phase of dynamic CT shows hypovascular nodule (arrow). c Delayed phase of dynamic CT shows

\section{CTNNB-1 mutated HCC}

CTNNB-1 (which encodes beta catenin) mutated HCC was estimated to account for about $30-40 \%$ of all HCCs [84]. CTNNB-1 mutated HCC is characterized by welldifferentiated tumors with cholestasis, microtrabecular and pseudoglandular patterns, lack of inflammatory infiltrates, low AFP expression [16] and a relatively favorable prognosis [85].

CTNNB-1 mutated HCC showed higher enhancement ratios on $\mathrm{HB}$ phase of gadoxetic acid-enhanced MRI with overexpression of OATP1B3 and a high ADC on DWI radiologically [73] (Fig. 8). Most CTNNB-1 mutated HCCs with hypoattenuating nodule (arrow). d This case was pathologically diagnosed to be a poorly differentiated HCC with positive of CK19 immunoactivity. (original magnification $\times 100$ )

a better prognosis are suspected to correspond to $\mathrm{HB}$ phase hyperintense $\mathrm{HCC}$ as mentioned above.

\section{Summary}

As outlined above, there are various factors and their imaging biomarkers are predictive of the biological nature of HCC. Imaging biomarkers suggesting less aggressive (better prognosis) or aggressive (worse prognosis) of HCC are summarized in Table 2.

The imaging biomarkers suggesting a less aggressive HCC nature are smaller size, solitary tumor, smooth margin 

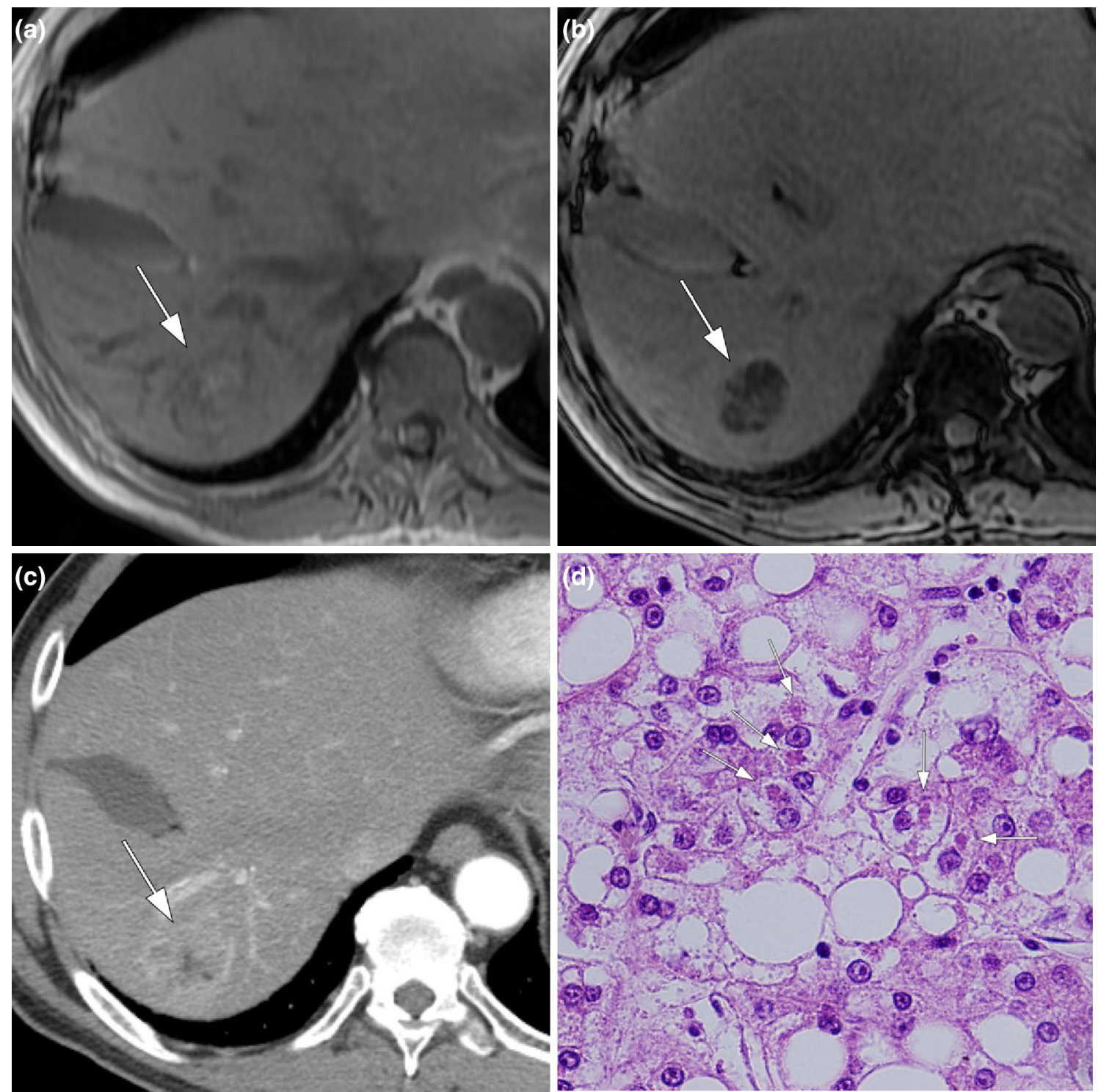

Fig. 11 Steatohepatitic HCC SH-HCC (Mod) in 65 year old male with $\mathrm{CH}$ (NASH). a In phase of T1-weighted image shows slightly hyperintense nodule (arrow). b Out of phase of T1-weighted image shows diffuse signal dropout of the nodule (arrow). c Arterial dominant phase of dynamic CT shows heterogeneous enhancement

suggesting small nodular type with indistinct margin ( $\mathrm{SN}$ IM) and simple nodular type with distinct margin (SN-DM), fibrous capsule or pseudocapsule, imaging biomarkers predicting early or well-differentiated grade, intra-tumoral-fat detection and low accumulation of fluorodeoxyglucose (FDG). On the other hand, the imaging biomarkers suggesting an aggressive HCC nature are larger size, multifocality, non-smooth margin suggesting simple nodular type with extranodular growth (SN-EG), confluent multinodular $(\mathrm{CMN})$, and infiltrative type, imaging biomarkers predicting poor differentiation, macrovascular thrombus, predictive of microvascular invasion imaging biomarkers, bile duct (arrow). d HE staining shows this HCC has large droplet steatosis, ballooning of tumor cells, Mallory-Denk body (arrows), and pericellular fibrosis. This HCC was confirmed to be SH-HCC. (original magnification $\times 400$ )

dilatation or thrombus, and high accumulation of FDG. In the genome-based molecular classification, CTNNB-1 mutated HCC shows a less aggressive biological nature, whereas CK19/EpCAM positive HCC and MTM-HCC show an aggressive biological nature.

The biological nature of HCC is not a simple issue that can be predicted with a single imaging biomarker, and it is thought that various imaging biomarkers are related. Currently, it is still difficult to comprehensively evaluate these imaging biomarkers and predict biological characteristics in total. In addition, there are other clinical biomarkers such as tumor markers that predict biological properties. Therefore, 
Table 2 Summary of the imaging biomarkers and molecular classification predicting the biological nature of hepatocellular carcinoma

\begin{tabular}{|c|c|c|}
\hline $\begin{array}{l}\text { Factors related with bio- } \\
\text { logical nature of HCC }\end{array}$ & Less aggressive (better prognostic) & Aggressive (worse prognostic) \\
\hline Size & Smaller & Larger \\
\hline Multi-focality & Solitaly & Multiple \\
\hline Macroscopic morphology & SN-IM/SN-DM/Capsule & SN-EG/CMN/Infiltrative type \\
\hline Grade of differentiation $^{\mathrm{a}}$ & Early/Well ${ }^{\mathrm{a}}$ & poorly $^{\mathrm{a}}$ \\
\hline Macrovascular invasion & & Macrovascular thrombus (+) \\
\hline Microvascular invasion & & $\begin{array}{l}\text { Non-smooth margin/ } \\
\text { Irregular circumferential enhancement/ } \\
\text { Peritumoral hypointensity on HB phase/ } \\
\text { Lower ADC/ } \\
\text { Larger size/ } \\
\text { Arterial rim enhancement/ } \\
\text { High accumulation of FDG/ } \\
\text { The disruption of the capsule/ } \\
\text { Multifocality }\end{array}$ \\
\hline Bile duct invasion & & Bile duct dilatation or thrombus \\
\hline Intra-tumoral fat & Intra-tumoral fat detection $(+)$ & \\
\hline Molecular factors & $\begin{array}{l}\text { Low accumulation of FDG/ } \\
\text { HB phase hyperintense HCC }\end{array}$ & High accumulation of FDG \\
\hline Molecular classification & CTNNB-1 mutated HCC & CK19/EpCAM positive HCC/MTM-HCC \\
\hline
\end{tabular}

${ }^{\text {a }}$ See the summary of the imaging biomarkers predicting the grade of differentiation in HCC (Fig. 6) it will be necessary in the future to establish a comprehensive evaluation system of the biological characteristics of HCC including various biomarkers predicting the prognosis so as to select the most appropriate treatment for individual HCC patient.

In conclusion, the biological nature of $\mathrm{HCC}$ is correlated with various factors, and an understanding of their imaging biomarkers can contribute to determining the most appropriate treatment plan of HCC. Genome-based molecular HCC classification has the possibility of contributing to future target therapy and personalized care. In the genome-based molecular HCC classification, the establishment of imaging biomarkers is not yet sufficient, although it is anticipated that this will be achieved in the near future.

Funding This work received no grant funding.

\section{Compliance with ethical standards}

Conflict of interest The authors have no conflict of interest to disclose with respect to this article.

Ethical statement This article does not contain any studies with human participants or animals performed by any of the authors.

\section{References}

1. Martins A, Cortez-Pinto H, Marques-Vidal P, Mendes N, Silva S, Fatela N, et al. Treatment and prognostic factors in patients with hepatocellular carcinoma. Liver Int. 2006. https://doi.org/10.111 1/j.1478-3231.2006.001285.x.

2. Shimada M, Rikimaru T, Hamatsu T, Yamashita Y, Terashi T, Taguchi K, et al. The role of macroscopic classification in nodulartype hepatocellular carcinoma. Am J Surg. 2001;182(2):177-82.

3. Lee CS, Hwang LY, Beasley RP, Hsu HC, Lee HS, Lin TY. Prognostic significance of histologic findings in resected small hepatocellular carcinoma. Acta Chir Scand. 1988;154(3):199-203.

4. Lai EC, Ng IO, Ng MM, Lok AS, Tam PC, Fan ST, et al. Longterm results of resection for large hepatocellular carcinoma: a multivariate analysis of clinicopathological features. Hepatology. 1990;11(5):815-8.

5. Oishi K, Itamoto T, Amano H, Fukuda S, Ohdan H, Tashiro H, et al. Clinicopathologic features of poorly differentiated hepatocellular carcinoma. J Surg Oncol. 2007. https://doi.org/10.1002/ jso.20661.

6. Jonas S, Bechstein WO, Steinmuller T, Herrmann M, Radke $\mathrm{C}$, Berg T, et al. Vascular invasion and histopathologic grading determine outcome after liver transplantation for hepatocellular carcinoma in cirrhosis. Hepatology. 2001. https://doi.org/10.1053/ jhep.2001.23561.

7. Shin WY, Suh KS, Lee HW, Kim J, Kim T, Yi NJ, et al. Prognostic factors affecting survival after recurrence in adult living donor liver transplantation for hepatocellular carcinoma. Liver Transpl. 2010. https://doi.org/10.1002/lt.22047.

8. Cha C, Fong Y, Jarnagin WR, Blumgart LH, DeMatteo RP. Predictors and patterns of recurrence after resection of hepatocellular carcinoma. J Am Coll Surg. 2003. https://doi.org/10.1016/j.jamco 1lsurg.2003.07.003.

9. Sumie S, Kuromatsu R, Okuda K, Ando E, Takata A, Fukushima $\mathrm{N}$, et al. Microvascular invasion in patients with hepatocellular carcinoma and its predictable clinicopathological factors. Ann Surg Oncol. 2008. https://doi.org/10.1245/s10434-008-9846-9.

10. Suh YG, Kim DY, Han KH, Seong J. Effective biliary drainage and proper treatment improve outcomes of hepatocellular carcinoma with obstructive jaundice. Gut Liver. 2014. https://doi. org/10.5009/gnl13370. 
11. Siripongsakun S, Lee JK, Raman SS, Tong MJ, Sayre J, Lu DS. MRI detection of intratumoral fat in hepatocellular carcinoma: potential biomarker for a more favorable prognosis. AJR Am J Roentgenol. 2012. https://doi.org/10.2214/ajr.12.8632.

12. Sun DW, An L, Wei F, Mu L, Shi XJ, Wang CL, et al. Prognostic significance of parameters from pretreatment (18)F-FDG PET in hepatocellular carcinoma: a meta-analysis. Abdom Radiol (NY). 2016. https://doi.org/10.1007/s00261-015-0603-9.

13. Kitao A, Matsui O, Yoneda N, Kozaka K, Kobayashi S, Koda W, et al. Hypervascular hepatocellular carcinoma: correlation between biologic features and signal intensity on gadoxetic acidenhanced MR images. Radiology. 2012. https://doi.org/10.1148/ radiol.12120226.

14. Hoshida Y, Toffanin S, Lachenmayer A, Villanueva A, Minguez B, Llovet JM. Molecular classification and novel targets in hepatocellular carcinoma: recent advancements. Semin Liver Dis. 2010. https://doi.org/10.1055/s-0030-1247131.

15. Goossens N, Sun X, Hoshida Y. Molecular classification of hepatocellular carcinoma: potential therapeutic implications. Hepat Oncol. 2015. https://doi.org/10.2217/hep.15.26.

16. Calderaro J, Couchy G, Imbeaud S, Amaddeo G, Letouze E, Blanc JF, et al. Histological subtypes of hepatocellular carcinoma are related to gene mutations and molecular tumour classification. $\mathbf{J}$ Hepatol. 2017. https://doi.org/10.1016/j.jhep.2017.05.014.

17. Pawlik TM, Delman KA, Vauthey JN, Nagorney DM, Ng IO, Ikai I, et al. Tumor size predicts vascular invasion and histologic grade: Implications for selection of surgical treatment for hepatocellular carcinoma. Liver Transpl. 2005. https://doi.org/10.1002/lt.20472.

18. Efremidis SC, Hytiroglou P, Matsui O. Enhancement patterns and signal-intensity characteristics of small hepatocellular carcinoma in cirrhosis: pathologic basis and diagnostic challenges. Eur Radiol. 2007. https://doi.org/10.1007/s00330-007-0705-z.

19. Kojiro M. Histopathology of liver cancers. Best Pract Res Clin Gastroenterol. 2005. https://doi.org/10.1016/j.bpg.2004.10.007.

20. Roskams T, Kojiro M. Pathology of early hepatocellular carcinoma: conventional and molecular diagnosis. Semin Liver Dis. 2010. https://doi.org/10.1055/s-0030-1247129.

21. Nakashima O, Sugihara S, Kage M, Kojiro M. Pathomorphologic characteristics of small hepatocellular carcinoma: a special reference to small hepatocellular carcinoma with indistinct margins. Hepatology. 1995;22(1):101-5.

22. Chandarana H, Robinson E, Hajdu CH, Drozhinin L, Babb JS, Taouli B. Microvascular invasion in hepatocellular carcinoma: is it predictable with pretransplant MRI? AJR Am J Roentgenol. 2011. https://doi.org/10.2214/ajr.10.4720.

23. Wang J, Li Q, Sun Y, Zheng H, Cui Y, Li H, et al. Clinicopathologic features between multicentric occurence and intrahepatic metastasis of multiple hepatocellular carcinomas related to HBV. Surg Oncol. 2009. https://doi.org/10.1016/j.suronc.2008.05.009.

24. Eggel H. Über das primäre Carcinom der Leber. Beitr Pathol Anat. 1901;30:506-604 (in German).

25. Liver Cancer Study Group of Japan. The general rules for the clinical and pathological study of primary liver cancer. 6th ed. Tokyo: Kanehara; 2015.

26. Hui AM, Takayama T, Sano K, Kubota K, Akahane M, Ohtomo $\mathrm{K}$, et al. Predictive value of gross classification of hepatocellular carcinoma on recurrence and survival after hepatectomy. J Hepatol. 2000;33(6):975-9.

27. He J, Shi J, Fu X, Mao L, Zhou T, Qiu Y, et al. The Clinicopathologic and Prognostic Significance of Gross Classification on Solitary Hepatocellular Carcinoma After Hepatectomy. Medicine (Baltim). 2015. https://doi.org/10.1097/md.0000000000001331.

28. Hatanaka K, Chung H, Kudo M, Haji S, Minami Y, Maekawa K, et al. Usefulness of the post-vascular phase of contrast-enhanced ultrasonography with sonazoid in the evaluation of gross types of hepatocellular carcinoma. Oncology. 2010. https://doi. org/10.1159/000315231.

29. Fujinaga Y, Kadoya M, Kozaka K, Shinmura R, Matsui O, Takayama T, et al. Prediction of macroscopic findings of hepatocellular carcinoma on hepatobiliary phase of gadolinium-ethoxybenzyldiethylenetriamine pentaacetic acid-enhanced magnetic resonance imaging: Correlation with pathology. Hepatol Res. 2013. https:// doi.org/10.1111/j.1872-034x.2012.01089.x.

30. Lim JH, Choi D, Park CK, Lee WJ, Lim HK. Encapsulated hepatocellular carcinoma: CT-pathologic correlations. Eur Radiol. 2006. https://doi.org/10.1007/s00330-006-0203-8.

31. Ros PR, Murphy BJ, Buck JL, Olmedilla G, Goodman Z. Encapsulated hepatocellular carcinoma: radiologic findings and pathologic correlation. Gastrointest Radiol. 1990;15(3):233-7.

32. Ishigami K, Yoshimitsu K, Nishihara Y, Irie H, Asayama Y, Tajima T, et al. Hepatocellular carcinoma with a pseudocapsule on gadolinium-enhanced MR images: correlation with histopathologic findings. Radiology. 2009. https://doi.org/10.1148/radio 1.2501071702 .

33. Kadoya M, Matsui O, Takashima T, Nonomura A. Hepatocellular carcinoma: correlation of MR imaging and histopathologic findings. Radiology. 1992. https://doi.org/10.1148/radio logy.183.3.1316622.

34. Grazioli L, Olivetti L, Fugazzola C, Benetti A, Stanga C, Dettori $\mathrm{E}$, et al. The pseudocapsule in hepatocellular carcinoma: correlation between dynamic MR imaging and pathology. Eur Radiol. 1999. https://doi.org/10.1007/s003300050629.

35. Hayashi M, Matsui O, Ueda K, Kawamori Y, Kadoya M, Yoshikawa J, et al. Correlation between the blood supply and grade of malignancy of hepatocellular nodules associated with liver cirrhosis: evaluation by CT during intraarterial injection of contrast medium. AJR Am J Roentgenol. 1999. https://doi.org/10.2214/ ajr.172.4.10587130.

36. Matsui O, Gabata T, Kobayashi S, Terayama N, Sanada J, Kouda $\mathrm{W}$, et al. Imaging of multistep human hepatocarcinogenesis. Hepatol Res. 2007. https://doi.org/10.1111/j.1872-034x.2007.00185.x.

37. Asayama Y, Yoshimitsu K, Nishihara Y, Irie H, Aishima S, Taketomi A, et al. Arterial blood supply of hepatocellular carcinoma and histologic grading: radiologic-pathologic correlation. AJR Am J Roentgenol. 2008. https://doi.org/10.2214/ajr.07.2117.

38. Kitao A, Matsui O, Yoneda N, Kozaka K, Shinmura R, Koda W, et al. The uptake transporter OATP8 expression decreases during multistep hepatocarcinogenesis: correlation with gadoxetic acid enhanced MR imaging. Eur Radiol. 2011. https://doi.org/10.1007/ s00330-011-2165-8.

39. Imai Y, Murakami T, Yoshida S, Nishikawa M, Ohsawa M, Tokunaga K, et al. Superparamagnetic iron oxide-enhanced magnetic resonance images of hepatocellular carcinoma: correlation with histological grading. Hepatology. 2000. https://doi.org/10.1053/ jhep.2000.9113.

40. Arita J, Hasegawa K, Takahashi M, Hata S, Shindoh J, Sugawara $\mathrm{Y}$, et al. Correlation between contrast-enhanced intraoperative ultrasound using Sonazoid and histologic grade of resected hepatocellular carcinoma. AJR Am J Roentgenol. 2011. https://doi. org/10.2214/ajr.10.4310.

41. Enomoto S, Tamai H, Shingaki N, Mori Y, Moribata K, Shiraki T, et al. Assessment of hepatocellular carcinomas using conventional magnetic resonance imaging correlated with histological differentiation and a serum marker of poor prognosis. Hepatol Int. 2011. https://doi.org/10.1007/s12072-010-9245-8.

42. Ebara M, Fukuda H, Kojima Y, Morimoto N, Yoshikawa M, Sugiura N, et al. Small hepatocellular carcinoma: relationship of signal intensity to histopathologic findings and metal content of the tumor and surrounding hepatic parenchyma. Radiology. 1999. https://doi.org/10.1148/radiology.210.1.r99ja4181. 
43. Yu JS, Lee JH, Park MS, Kim KW. Hyperintense nodules on nonenhanced T1-weighted gradient-echo magnetic resonance imaging of cirrhotic liver: fate and clinical implications. J Magn Reson Imaging. 2006. https://doi.org/10.1002/jmri.20674.

44. Nakanishi M, Chuma M, Hige S, Omatsu T, Yokoo H, Nakanishi $\mathrm{K}$, et al. Relationship between diffusion-weighted magnetic resonance imaging and histological tumor grading of hepatocellular carcinoma. Ann Surg Oncol. 2012. https://doi.org/10.1245/s1043 4-011-2066-8.

45. Nishie A, Tajima T, Asayama Y, Ishigami K, Kakihara D, Nakayama T, et al. Diagnostic performance of apparent diffusion coefficient for predicting histological grade of hepatocellular carcinoma. Eur J Radiol. 2011. https://doi.org/10.1016/j.ejrad .2010.06.019.

46. Izuishi K, Yamamoto Y, Mori H, Kameyama R, Fujihara S, Masaki T, et al. Molecular mechanisms of [18F]fluorodeoxyglucose accumulation in liver cancer. Oncol Rep. 2014. https://doi. org/10.3892/or.2013.2886.

47. Llovet JM, Fuster J, Bruix J, Barcelona-Clinic Liver Cancer G. The Barcelona approach: diagnosis, staging, and treatment of hepatocellular carcinoma. Liver Transpl. 2004. https://doi. org/10.1002/lt.20034.

48. Kanai T, Hirohashi S, Upton MP, Noguchi M, Kishi K, Makuuchi $\mathrm{M}$, et al. Pathology of small hepatocellular carcinoma. A proposal for a new gross classification. Cancer. 1987;60(4):810-9.

49. Edmondson HA, Steiner PE. Primary carcinoma of the liver: a study of 100 cases among 48,900 necropsies. Cancer. 1954;7(3):462-503.

50. Chou CT, Chen RC, Lin WC, Ko CJ, Chen CB, Chen YL. Prediction of microvascular invasion of hepatocellular carcinoma: preoperative CT and histopathologic correlation. AJR Am J Roentgenol. 2014. https://doi.org/10.2214/ajr.13.10595.

51. Ariizumi S, Kitagawa K, Kotera Y, Takahashi Y, Katagiri S, Kuwatsuru R, et al. A non-smooth tumor margin in the hepatobiliary phase of gadoxetic acid disodium (Gd-EOB-DTPA)enhanced magnetic resonance imaging predicts microscopic portal vein invasion, intrahepatic metastasis, and early recurrence after hepatectomy in patients with hepatocellular carcinoma. J Hepato-biliary-pancreat Sci. 2011. https://doi.org/10.1007/s0053 4-010-0369-y.

52. Kim H, Park MS, Choi JY, Park YN, Kim MJ, Kim KS, et al. Can microvessel invasion of hepatocellular carcinoma be predicted by pre-operative MRI? Eur Radiol. 2009. https://doi.org/10.1007/ s00330-009-1331-8.

53. Nishie A, Yoshimitsu K, Asayama Y, Irie H, Tajima T, Hirakawa $\mathrm{M}$, et al. Radiologic detectability of minute portal venous invasion in hepatocellular carcinoma. AJR Am J Roentgenol. 2008. https ://doi.org/10.2214/ajr.07.2810.

54. Kim KA, Kim MJ, Jeon HM, Kim KS, Choi JS, Ahn SH, et al. Prediction of microvascular invasion of hepatocellular carcinoma: usefulness of peritumoral hypointensity seen on gadoxetate disodium-enhanced hepatobiliary phase images. J Magn Reson Imaging. 2012. https://doi.org/10.1002/jmri.22876.

55. Nishie A, Asayama Y, Ishigami K, Kakihara D, Nakayama T, Ushijima Y, et al. Clinicopathological significance of the peritumoral decreased uptake area of gadolinium ethoxybenzyl diethylenetriamine pentaacetic acid in hepatocellular carcinoma. J Gastroenterol Hepatol. 2014. https://doi.org/10.1111/jgh.12423.

56. Suh YJ, Kim MJ, Choi JY, Park MS, Kim KW. Preoperative prediction of the microvascular invasion of hepatocellular carcinoma with diffusion-weighted imaging. Liver Transpl. 2012. https://doi. org/10.1002/lt.23502.

57. Ahn SY, Lee JM, Joo I, Lee ES, Lee SJ, Cheon GJ, et al. Prediction of microvascular invasion of hepatocellular carcinoma using gadoxetic acid-enhanced MR and (18)F-FDG PET/CT. Abdom Imaging. 2015. https://doi.org/10.1007/s00261-014-0256-0.
58. Hirokawa F, Hayashi M, Miyamoto Y, Asakuma M, Shimizu $\mathrm{T}$, Komeda K, et al. Outcomes and predictors of microvascular invasion of solitary hepatocellular carcinoma. Hepatol Res. 2014. https://doi.org/10.1111/hepr.12196.

59. An C, Kim DW, Park YN, Chung YE, Rhee H, Kim MJ. Single Hepatocellular carcinoma: preoperative MR imaging to predict early recurrence after curative resection. Radiology. 2015. https ://doi.org/10.1148/radiol.15142394.

60. Kierans AS, Leonardou P, Hayashi P, Brubaker LM, Elazzazi M, Shaikh F, et al. MRI findings of rapidly progressive hepatocellular carcinoma. Magn Reson Imaging. 2010. https://doi. org/10.1016/j.mri.2010.03.005.

61. Kawamura Y, Ikeda K, Hirakawa M, Yatsuji H, Sezaki H, Hosaka T, et al. New classification of dynamic computed tomography images predictive of malignant characteristics of hepatocellular carcinoma. Hepatol Res. 2010. https://doi. org/10.1111/j.1872-034x.2010.00703.x.

62. Kornberg A, Freesmeyer M, Barthel E, Jandt K, Katenkamp $\mathrm{K}$, Steenbeck J, et al. 18F-FDG-uptake of hepatocellular carcinoma on PET predicts microvascular tumor invasion in liver transplant patients. Am J Transpl. 2009. https://doi.org/10.111 1/j.1600-6143.2008.02516.x.

63. Lee S, Kim SH, Lee JE, Sinn DH, Park CK. Preoperative gadoxetic acid-enhanced MRI for predicting microvascular invasion in patients with single hepatocellular carcinoma. J Hepatol. 2017. https://doi.org/10.1016/j.jhep.2017.04.024.

64. Kojiro M, Kawabata K, Kawano Y, Shirai F, Takemoto N, Nakashima T. Hepatocellular carcinoma presenting as intrabile duct tumor growth: a clinicopathologic study of 24 cases. Cancer. 1982;49(10):2144-7.

65. Navadgi S, Chang CC, Bartlett A, McCall J, Pandanaboyana S. Systematic review and meta-analysis of outcomes after liver resection in patients with hepatocellular carcinoma (HCC) with and without bile duct thrombus. HPB (Oxf). 2016. https://doi. org/10.1016/j.hpb.2015.12.003.

66. Yu XH, Xu LB, Liu C, Zhang R, Wang J. Clinicopathological characteristics of 20 cases of hepatocellular carcinoma with bile duct tumor thrombi. Dig Dis Sci. 2011. https://doi.org/10.1007/ s10620-010-1256-8.

67. Hu J, Pi Z, Yu MY, Li Y, Xiong S. Obstructive jaundice caused by tumor emboli from hepatocellular carcinoma. Am Surg. 1999;65(5):406-10.

68. Kutami R, Nakashima Y, Nakashima O, Shiota K, Kojiro M. Pathomorphologic study on the mechanism of fatty change in small hepatocellular carcinoma of humans. J Hepatol. 2000;33(2):282-9.

69. Asayama Y, Nishie A, Ishigami K, Ushijima Y, Takayama Y, Okamoto D, et al. Fatty change in moderately and poorly differentiated hepatocellular carcinoma on MRI: a possible mechanism related to decreased arterial flow. Clin Radiol. 2016. https ://doi.org/10.1016/j.crad.2016.04.020.

70. Min JH, Kim YK, Lim S, Jeong WK, Choi D, Lee WJ. Prediction of microvascular invasion of hepatocellular carcinomas with gadoxetic acid-enhanced MR imaging: Impact of intratumoral fat detected on chemical-shift images. Eur J Radiol. 2015. https://doi.org/10.1016/j.ejrad.2015.03.002.

71. Kitao A, Zen Y, Matsui O, Gabata T, Kobayashi S, Koda W, et al. Hepatocellular carcinoma: signal intensity at gadoxetic acid-enhanced MR Imaging-correlation with molecular transporters and histopathologic features. Radiology. 2010. https:// doi.org/10.1148/radiol.10092214.

72. Narita M, Hatano E, Arizono S, Miyagawa-Hayashino A, Isoda $\mathrm{H}$, Kitamura K, et al. Expression of OATP1B3 determines uptake of Gd-EOB-DTPA in hepatocellular carcinoma. J Gastroenterol. 2009;44(7):793-8. 
73. Kitao A, Matsui O, Yoneda N, Kozaka K, Kobayashi S, Sanada $\mathrm{J}$, et al. Hepatocellular Carcinoma with beta-Catenin Mutation: Imaging and Pathologic Characteristics. Radiology. 2015. https ://doi.org/10.1148/radiol.14141315.

74. Yoneda N, Matsui O, Kitao A, Kita R, Kozaka K, Koda W, et al. Hypervascular hepatocellular carcinomas showing hyperintensity on hepatobiliary phase of gadoxetic acid-enhanced magnetic resonance imaging: a possible subtype with mature hepatocyte nature. Jpn J Radiol. 2013. https://doi.org/10.1007/s11604-013-0224-6.

75. Yamashita T, Kitao A, Matsui O, Hayashi T, Nio K, Kondo M, et al. Gd-EOB-DTPA-enhanced magnetic resonance imaging and alpha-fetoprotein predict prognosis of early-stage hepatocellular carcinoma. Hepatology. 2014. https://doi.org/10.1002/hep.27093.

76. Ning BF, Ding J, Yin C, Zhong W, Wu K, Zeng X, et al. Hepatocyte nuclear factor 4 alpha suppresses the development of hepatocellular carcinoma. Cancer Res. 2010. https://doi. org/10.1158/0008-5472.can-10-0824.

77. Uenishi T, Kubo S, Yamamoto T, Shuto T, Ogawa M, Tanaka $\mathrm{H}$, et al. Cytokeratin 19 expression in hepatocellular carcinoma predicts early postoperative recurrence. Cancer Sci. 2003;94(10):851-7.

78. Chung GE, Lee JH, Yoon JH, Myung SJ, Lee K, Jang JJ, et al. Prognostic implications of tumor vascularity and its relationship to cytokeratin 19 expression in patients with hepatocellular carcinoma. Abdom Imaging. 2012. https://doi.org/10.1007/s0026 1-011-9756-3.

79. Choi SY, Kim SH, Park CK, Min JH, Lee JE, Choi YH, et al. Imaging features of gadoxetic acid-enhanced and diffusionweighted MR imaging for identifying cytokeratin 19-positive hepatocellular carcinoma: a retrospective observational study. Radiology. 2018. https://doi.org/10.1148/radiol.2017162846.

80. Breuhahn K, Baeuerle PA, Peters M, Prang N, Tox U, KohneVolland R, et al. Expression of epithelial cellular adhesion molecule (Ep-CAM) in chronic (necro-)inflammatory liver diseases and hepatocellular carcinoma. Hepatol Res. 2006. https:// doi.org/10.1016/j.hepres.2005.10.006.

81. Yamashita T, Forgues M, Wang W, Kim JW, Ye Q, Jia H, et al. EpCAM and alpha-fetoprotein expression defines novel prognostic subtypes of hepatocellular carcinoma. Cancer Res. 2008. https:// doi.org/10.1158/0008-5472.can-07-6013.

82. Yamashita T, Ji J, Budhu A, Forgues M, Yang W, Wang HY, et al. EpCAM-positive hepatocellular carcinoma cells are tumor-initiating cells with stem/progenitor cell features. Gastroenterology. 2009. https://doi.org/10.1053/j.gastro.2008.12.004.

83. Salomao M, Yu WM, Brown RS Jr, Emond JC, Lefkowitch JH. Steatohepatitic hepatocellular carcinoma (SH-HCC): a distinctive histological variant of HCC in hepatitis C virus-related cirrhosis with associated NAFLD/NASH. Am J Surg Pathol. 2010. https:// doi.org/10.1097/pas.0b013e3181f31caa.

84. Audard V, Grimber G, Elie C, Radenen B, Audebourg A, Letourneur F, et al. Cholestasis is a marker for hepatocellular carcinomas displaying beta-catenin mutations. J Pathol. 2007. https ://doi.org/10.1002/path.2169.

85. Mao TL, Chu JS, Jeng YM, Lai PL, Hsu HC. Expression of mutant nuclear beta-catenin correlates with non-invasive hepatocellular carcinoma, absence of portal vein spread, and good prognosis. J Pathol. 2001. https://doi.org/10.1002/10969896(2000)9999:9999\%3c:aid-path720\%3e3.0.co;2-3.

Publisher's Note Springer Nature remains neutral with regard to jurisdictional claims in published maps and institutional affiliations. 